\title{
Power-efficient distributed resource allocation under goodput QoS constraints for heterogeneous networks
}

\author{
Riccardo Andreotti ${ }^{1,2 *}$, Paolo Del Fiorentino ${ }^{1}$, Filippo Giannetti ${ }^{1}$ and Vincenzo Lottici ${ }^{1}$ (D)
}

\begin{abstract}
This work proposes a distributed resource allocation (RA) algorithm for packet bit-interleaved coded OFDM transmissions in the uplink of heterogeneous networks (HetNets), characterized by small cells deployed over a macrocell area and sharing the same band. Every user allocates its transmission resources, i.e., bits per active subcarrier, coding rate, and power per subcarrier, to minimize the power consumption while both guaranteeing a target quality of service (QoS) and accounting for the interference inflicted by other users transmitting over the same band. The QoS consists of the number of information bits delivered in error-free packets per unit of time, or goodput (GP), estimated at the transmitter by resorting to an efficient effective SNR mapping technique. First, the RA problem is solved in the point-to-point case, thus deriving an approximate yet accurate closed-form expression for the power allocation (PA). Then, the interference-limited HetNet case is examined, where the RA problem is described as a non-cooperative game, providing a solution in terms of generalized Nash equilibrium. Thanks to the closed-form of the PA, the solution analysis is based on the best response concept. Hence, sufficient conditions for existence and uniqueness of the solution are analytically derived, along with a distributed algorithm capable of reaching the game equilibrium.
\end{abstract}

Keywords: Power-efficiency, Game theory, Goodput

\section{Introduction}

Future wireless networks are required to offer extremely enhanced capabilities including very high achievable data rates, very low latency, ultra-high reliability, and the possibility to handle very high density of devices [1]. On the other hand, since this trend dramatically contributes to the pollution related to energy consumption [2], energyefficient wireless communications emerged as a viable design concept to reduce the $\mathrm{CO}_{2}$ emission in the next years [3]. In addition, to manage the envisioned huge demand of traffic, a very promising solution is offered by the concept of heterogeneous network (HetNet), where small-cell (SC) networks, characterized by low-cost, lowpower, and low-coverage base stations (BSs), i.e., small BSs (SBSs), are massively deployed over the macrocell (MC) areas. Their adoption allows offloading the traffic of the

*Correspondence: riccardo.andreotti@wiser.it

'Department of Information Engineering, University of Pisa, Via G. Caruso 16, I-56122 Pisa, Italy

${ }^{2}$ Wireless Systems Engineering and Research (WISER) S.r.l., Via Fiume 23, |-57123 Livorno, Italy macro network, thus increasing the offered data rate and spectrum re-usage and providing, at the same time, a more power-efficient architecture thanks to their reduced coverage [4]. Since in HetNets SCs share the same frequency bands of the MC [5], one of the main technical effort to be made is the management of the interference, either between SC and MC users (cross-tier interference) or between users in different SCs (co-tier interference). Hence, the random deployment of SCs together with the heterogeneity of these networks naturally calls for decentralized resource allocation (RA) strategies [6], where every user, independently from the other ones, maximizes its performance taking into account interference experienced on its transmission band. Indeed, distributed RA strategies only require the knowledge of local channel state information (CSI), exchanged between each user and its associated BS, thereby avoiding the waste of energy associated with centralized algorithms based instead on a huge information exchange between the users and/or the network administrator $[6,7]$. 


\subsection{Related works}

Most of the works on RA in HetNets focus on the improvement of the energy efficiency (EE) as in [8], where the authors study the RA problem optimizing the EE over the downlink of an orthogonal frequency division multiplexing (OFDM) cognitive radio (CR) HetNet. A joint subcarrier allocation (SA) and power allocation (PA) solution is provided to guarantee quality of service (QoS) requirements for both secondary and primary users in the cognitive HetNet. Moreover, the authors propose a lowcomplexity RA algorithm in presence of imperfect CSI. In [9], authors address a joint EE RA and interference alignment problem for downlink multiple-input multipleoutput (MIMO) transmissions in HetNets. The EE maximization problem is solved in order to provide time-slot allocation, PA, and beamforming. Unlike [8, 9], paper [10] analyzes RA techniques with different levels of CSI for uplink orthogonal frequency-division multiple access (OFDMA) transmissions in HetNets with one macrocell and cognitive small cells. Here, the purpose of the RA is to maximize the weighted sum of instantaneous rates of all the users, providing a joint PA and SA. Article [11] discusses possible future developments for HetNet in fifthgeneration (5G) communications, where massive MIMO and mmWave technologies may be included, emphasizing that RA solutions will once again be one of the most critical issues. Besides, in this work a distributed RA problem over interference channels is tackled.

The most suited framework to study this kind of problem is identified in the theory of non-cooperative games [12], as outlined in $[13,14]$ about the uplink power control (PC) problem for flat and frequency-selective channels, respectively. Since then, the literature on this topic has increased more and more. Worth to be cited are $[15,16]$, where the energy-efficient PC problem for wireless data and code division multiple access (CDMA) networks is addressed, respectively, $[17,18]$, wherein the NCG framework is exploited to tackle the distributed PA problem for EE maximization in OFDM channels and MIMO HetNets, respectively, whereas in [19] the issue of dynamic RA is investigated in the context of multi-user cognitive networks, by exploiting a NCG for signal waveform design combined with sparsity constraints. In [20-22], the equilibrium analysis of the NCG describing the rate maximization problem in multicarrier and MIMO channels is discussed. A minimum PA game over OFDM channels with rate constraints is proposed in [23], whereas the PA problem based on the EE maximization under rate constraints is studied for HetNets in [6]. A final interesting work is presented in [24], where the authors investigate the RA problem for device-to-device (D2D) uplink communications in MIMO cellular networks. The $\mathrm{RA}$ is evaluated by means of a non-cooperative game that provides a joint optimization of channel allocation, power control, and precoding of the D2D users, by maximizing the sum-rate of the cellular uplink network. The distributed RA solution in [24] is exploited in [25] for D2D communications in MIMO HetNets.

A common feature to all the above works is that they rely on the assumption of infinite-length Gaussian codebooks and thus employ the channel capacity as figure of merit. However, when dealing with real systems characterized by practical and finite-sized modulation and coding schemes and automatic repeat request (ARQ) mechanisms, such information theoretical performance metric may offer an unreliable picture of the actual link performance [26]. In this cases, a suitable performance measure is the number of information bits delivered in error-free packets per unit of time, named goodput (GP) for short [26,27]. To our best knowledge, GP-based distributed RA strategies have been addressed so far in few works only, as in [28, 29]. In [28], a PC strategy for CDMA ad hoc networks is proposed, aiming at maximizing the GP to power ratio under transmission rate and power constraints, whereas in [29], a network utility maximization problem with GP flow variables under queues stability constraints is proposed for flat fading mobile ad-hoc networks.

\subsection{Contributions}

This work proposes a novel distributed RA strategy for an energy-aware communication in the uplink of OFDM-based HetNets. Assuming a packet-oriented bit-interleaved coded (BIC) OFDM transmission with practical modulation and coding formats and ARQ mechanisms, power consumption of all users is minimized, provided they meet a given QoS in terms of a target GP value. In order to allocate the transmission resources according to the available CSI, namely, bit loading (BL) vector, i.e., bits per active subcarrier, coding rate, and PA vector, the transmitter requires an estimate of GP, called expected goodput (EGP). The analytical formulation of such performance metric under frequency-selective fading channel scenarios is efficiently obtained resorting to the link performance prediction (LPP) method known as effective signal-to-noise ratio mapping (ESM) [30] and, specifically, exploiting the ESM technique proposed in [31]. Thus, the distributed RA strategy allows every user to minimize its power consumption, meeting at the same time its QoS constraint expressed in terms of target EGP, by selfishly allocating its transmission resources. Hence, in view of all the above features (especially, the EGP evaluation for BICOFDM systems over frequency-selective fading channels), our work brings the following competitive contributions.

1) As first step, the RA problem is analyzed for the point-to-point case (P2P), i.e., for a single transmitter receiver pair without interference. The optimal solution as well as an approximate yet accurate 
closed-form one, which is reminiscent of the water-filling solution for Gaussian signaling, is derived for the PA.

2) The RA problem is then extended to the the interference-limited HetNet scenario. The PA problem is solved in distributed manner as a function of the $\mathrm{BL}$ vector and the coding rate, by modeling it as a NCG, where players, strategy, and the utility function correspond to users, PA vector, and power consumption, respectively. Due to the QoS constraints imposed on the GP level, the set of strategies of each user depends on the strategies of the other users. This is the basic reason why the solution is the generalized Nash equilibrium (GNE) [32]. To be more specific, the GNE is described by a set of fixed-point equations based on the best response (BR) strategy of each player, wherein the BR (depending on the target EGP level) is obtained by capitalizing on the water-filling-like solution to the PA. Sufficient conditions for the feasibility of the problem, as well as for the existence and uniqueness of the GNE, are analytically derived. Additionally, some insights on the relationship between the GNE and the pareto optimal (PO) solution are provided.

3) A distributed RA algorithm, that allows the network to reach the equilibrium, is proposed. To support our findings, the algorithm performance is corroborated by physical layer simulations of the HetNet over realistic wireless scenarios.

Notations. Matrices are in upper case bold while column vectors are in lower case bold; $[\cdot]^{\mathrm{T}}$ is the transpose of a matrix or a vector; $\lceil x\rceil$ denotes the nearest greater integer than $x$; $\times$ denotes the Cartesian product; calligraphic mathematical symbols, e.g., $\mathcal{A}$, represent sets; $|\mathcal{A}|$ denotes the cardinality of the set $\mathcal{A} ; \mathcal{A}(i)$ is the $i$ th element of the set $\mathcal{A} ; y=[x]_{a}^{b}$ means $y=x$ if $a<x<b, x=a(x=b)$ if $x \leq a(x \geq b) ; y=[x]^{+}$means $y=x(y=0)$ if $x>0$ $(x \leq 0)$; the square root of a vector $\mathbf{x}$ is intended as the vector including the square root entries of $\mathbf{x} ; \mathbf{a} \odot \mathbf{b}$ denotes the element-wise multiplication between $\mathbf{a}$ and $\mathbf{b}$; and $a \perp b$ means $a \cdot b=0$; inequalities between vectors are evaluated element-wise.

\section{HetNet model}

In this section, the HetNet scenario is first introduced and then the BIC-OFDM uplink channel with multiple access interference (MAI) is described.

\subsection{HetNet scenario description}

The HetNet scenario is depicted in Fig. 1 and is composed of $Q+1 \mathrm{BSs}$, whose indexes belong to the set $\mathcal{Q} \triangleq\{0, \cdots, Q\}$. In particular, there is one macro base station (MBS), with index $q=0$, and $Q$ SBSs, with indexes $q=1, \cdots, Q$, all of them sharing the same band $B$. Within the network, there exist $K$ users belonging to the set $\mathcal{K} \triangleq\{1, \cdots, K\}$, each of them transmitting to one of the BSs, so that, if $\mathcal{K}_{q}$ denotes the set of users served by BS $q$, then $\mathcal{K}=\mathcal{K}_{1} \cup \cdots \cup \mathcal{K}_{Q}$, with $\mathcal{K}_{q} \cap \mathcal{K}_{r}=\emptyset$ if $q \neq r$. The transmission signaling between every user and the corresponding BS is based on the OFDM format, i.e., the available bandwidth $B$ is subdivided into $N$ orthogonal subcarriers. Further, users within the same cell, either MC or $\mathrm{SC}$, transmits over orthogonal frequencies according

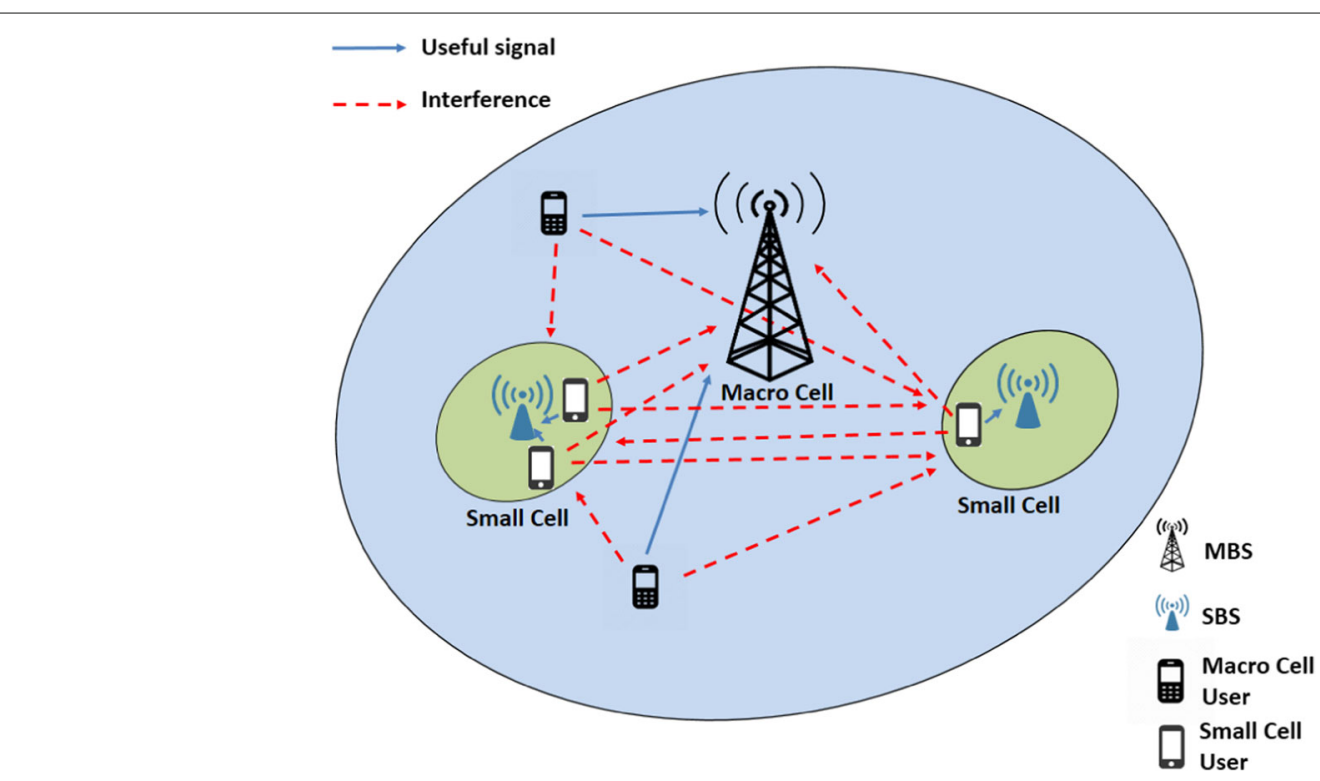

Fig. 1 Example of the HetNet scenario 
to the OFDMA scheme [3]. Though, since SCs and MC share the same band, the same subcarrier can be used at the same time by users served by either different SBSs or the MBS, leading to inter-cell interference (ICI). For the sake of notation, let us define the mapping $\phi: \mathcal{K} \rightarrow \mathcal{Q}$ to univocally associate each user $k \in \mathcal{K}$ to the relevant BS $q \in \mathcal{Q}$, so that $\phi(k)$, with $\phi(k)=q$, identifies the link between user $k$ and its $\mathrm{BS} q$.

\subsection{BIC-OFDM system description}

The block diagram of the uplink between user $k \in \mathcal{K}$ and its BS $\phi(k) \in \mathcal{Q}$ is depicted in Fig. 2. At the transmitter side, packets coming from the upper layers (usually IP packets) are processed as follows. First, each of them is one-to-one mapped to a radio link control sub-layer protocol data unit (RLC-PDU), composed of $U_{k}^{(\mathrm{i})}$ bits (containing the header, payload, and cyclic redundancy check). Then, the RLC-PDU is encoded producing a codeword of $U_{k}^{(\mathrm{s})} \triangleq U_{k}^{(\mathrm{i})} / r_{k}$ coded binary symbols (CBS), where $r_{k} \in \mathcal{D}_{r}$ is the coding rate and $\mathcal{D}_{r}$ the set of feasible coding rates. After bit-interleaving, the CBSs are Gray-mapped into $S_{k}$ complex-valued symbols, which are transmitted through a frame of $L_{k} \triangleq\left\lceil S_{k} / N_{k}\right\rceil$ consecutive OFDM blocks, where $N_{k} \triangleq\left|\mathcal{N}_{k}\right|$, being $\mathcal{N}_{k} \subseteq \mathcal{N} \triangleq\{1, \ldots, N\}$. The generic OFDM block, $\forall k \in \mathcal{K}$, consists of the vector $\mathbf{x}_{k} \triangleq\left[x_{k, 1}, \cdots, x_{k, N}\right]^{\mathrm{T}}$, where $x_{k, n}$ is a $2^{m_{k, n}}$-QAM unitaryenergy symbol, being $m_{k, n} \in \mathcal{D}_{m} \triangleq\left\{2, \cdots, m_{\max }\right\}$ the number of bits allocated on the $n$th subcarrier, if $n \in \mathcal{N}_{k}$, and $x_{k, n}=0$ if $n \notin \mathcal{N}_{k}$. In the following, we will assume, for the sake of simplicity, uniform bit loading (BL), that is, $m_{k, n}=m_{k} \in \mathcal{D}_{m}, \forall n \in \mathcal{N}_{k}$. The vector $\mathbf{x}_{k}$ is element-wise multiplied by the square root of the power allocation (PA) vector $\mathbf{p}_{k} \triangleq\left[p_{k, 1}, \cdots, p_{k, N}\right]^{\mathrm{T}}$, where $p_{k, n}>0$ if $n \in \mathcal{N}_{k}$ and $p_{k, n}=0$ if $n \notin \mathcal{N}_{k}$, such that the available power limit $P_{k}$ is satisfied according to

$$
\sum_{n \in \mathcal{N}_{k}} p_{k, n} \leq P_{k}, \quad \forall k \in \mathcal{K} .
$$

Subsequently, IFFT operation is performed, cyclic prefix $(\mathrm{CP})$ is inserted and after the digital-to-analog conversion, the OFDM signals of all the $K$ users active in both the $\mathrm{MC}$ and the SCs are each transmitted in uplink over (different) frequency-selective block-fading channels, which are assumed stationary for the whole packet transmission duration.

Focusing on the $k$ th user's signal at the $\phi(k)$ th $\mathrm{BS}, k \in \mathcal{K}$, at the FFT output, the received sample on subcarrier $n \in \mathcal{N}_{k}$ is

$$
z_{k, n}=\sqrt{p_{k, n}} h_{k, \phi}(k), n x_{k, n}+I_{k, n}+w_{k, n},
$$

where $h_{j, s, n}$ denotes the channel coefficient between user $j$ and BS $s$ over subcarrier $n, w_{k, n} \in \mathcal{N}\left(0, \sigma_{w_{k, n}}^{2}\right)$ is the zero-mean complex-valued Gaussian random variable modeling the ambient noise, and

$$
I_{k, n} \triangleq \sum_{\substack{j=1 \\ j \neq k}}^{K} \sqrt{p_{j, n}} h_{j, \phi(k), n} x_{j, n}
$$

represents the ICI, which is described as additive colored Gaussian noise with zero mean and variance

$$
\sigma_{I_{k, n}}^{2} \triangleq \sum_{\substack{j=1 \\ j \neq k}}^{K} p_{j, n}\left|h_{j, \phi(k), n}\right|^{2}, \quad \forall n \in \mathcal{N}_{k} .
$$

As for (3), we note that since users within the same cell $q \in \mathcal{Q}$ transmit according to the OFDMA scheme, then

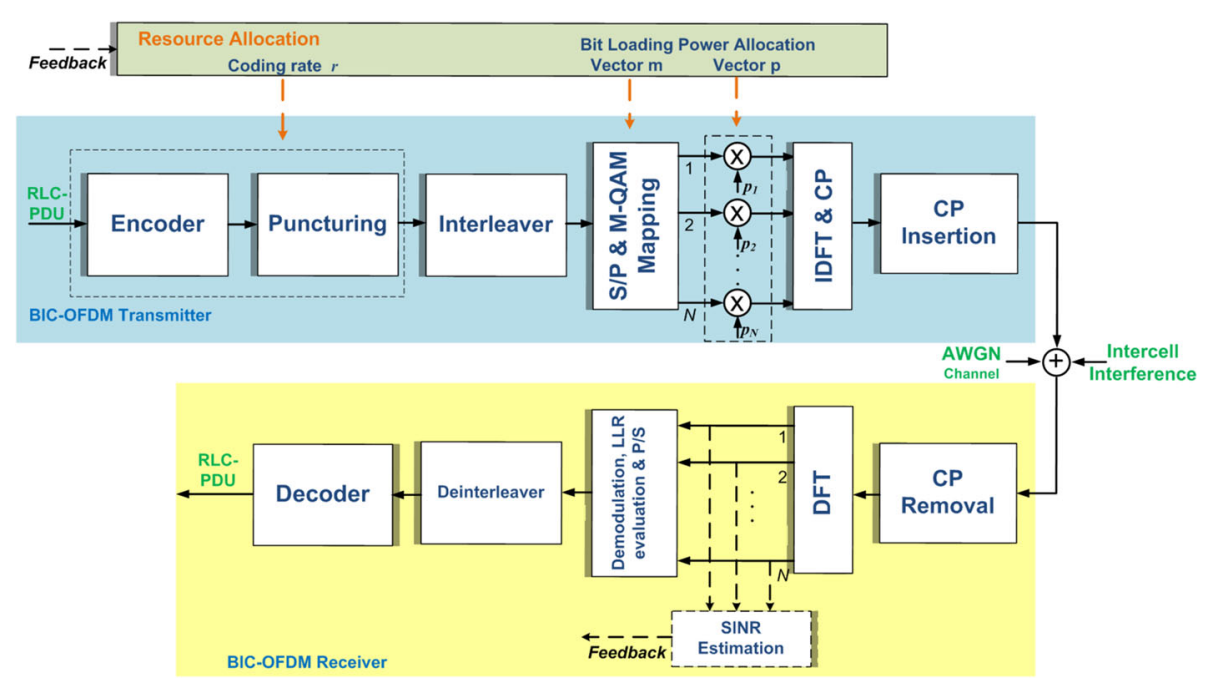

Fig. 2 BIC-OFDM communication link 
for a given subcarrier $n \in \mathcal{N}, p_{k, n}>0$ at most for one user $k \in \mathcal{K}$ such that $\phi(k)=q$, whereas $p_{i, n}=0$ for all the other users $i \in \mathcal{K}$ such that $\phi(i)=q$. Finally, the signal-to-interference-plus-noise ratio (SINR) vector evaluated for $k$ th user link results as $\boldsymbol{\Gamma}_{k}=\mathbf{p}_{k} \odot \boldsymbol{\gamma}_{k}$, where $\gamma_{k} \triangleq\left[\gamma_{k, 1}, \cdots, \gamma_{k, N}\right]^{\mathrm{T}}$, with

$$
\gamma_{k, n}=\left\{\begin{array}{cc}
\frac{\mid h_{k, \phi}(k), n}{\left.\right|^{2}} & \forall n \in \mathcal{N}_{k} \\
\sigma_{I_{k, n}}^{2}+\sigma_{w_{k, n}}^{2} & \forall n \notin \mathcal{N}_{k} \\
0 & \forall n .
\end{array}\right.
$$

\section{Performance metrics background and problem formulation}

In this section, a brief description of the adopted LPP metric is first given, then the optimization problem (OP) for minimum power consumption under EGP constraint satisfaction is introduced.

\subsection{LPP background}

As discussed in Section 1, practical modulation and coding schemes are employed; therefore, the GP metric is more suitable in giving a reliable picture of the actual link performance. Here, in order to apply the RA algorithm at the transmitter, the key issue is to get a prediction of the GP, i.e., the EGP, which in turn depends on the estimate of the link packet error rate (PER) $[28,31]$.

In a frequency-selective scenario as that considered in our paper, however, the expression of the PER may be hard to derive in closed-form. An efficient solution to pursue is given by the ESM techniques [30], which rely on one-toone mapping $\boldsymbol{\Gamma}_{k}$ into the scalar $\bar{\gamma}_{k}$, the so-called effective SNR (ESNR). Considering that a given subcarrier of every user $k \in \mathcal{K}$ can be loaded with $m \in \mathcal{D}_{m}$ bits, or switchedoff, i.e., $m=0$, let us denote with $\mathcal{D}_{\mathbf{m}}$ the set collecting the $\left|\mathcal{D}_{m}\right| \cdot 2^{N}$ possible bit allocation vectors. Hence, for a given transmission mode (TM) $\boldsymbol{\varphi}_{k} \triangleq\left\{r_{k}, \mathbf{m}_{k}\right\} \in \mathcal{D}_{r} \times \mathcal{D}_{\mathbf{m}}$, the ESNR $\bar{\gamma}_{k}$ is such that

$$
\operatorname{PER}_{\varphi_{k}}\left(\boldsymbol{\Gamma}_{k}\right)=\Phi_{r_{k}}\left(\bar{\gamma}_{k}\right)
$$

where $\operatorname{PER}_{\varphi_{k}}$ and $\Phi_{r_{k}}$ denote the PER of the coded BICOFDM system over frequency-selective channel employing TM $\varphi_{k}$ and that of the equivalent coded BPSK system over AWGN channel experiencing the ESNR $\bar{\gamma}_{k}$, respectively. Worth of being observed, $\Phi_{r_{k}}$, according to [33, 34], is an analytic, monotonically decreasing, and convex function in the region of interest.

Among the ESM techniques proposed in the literature, the $\kappa$ ESM method, based on the cumulant moment generating function of the log-likelihood metrics at the input of the soft decoder, is here exploited, since it offers a competitive accuracy-versus-complexity trade-off [31]. Herein, the ESNR $\bar{\gamma}_{k}$ is evaluated as $\bar{\gamma}_{k}\left(\boldsymbol{\varphi}_{k}, \mathbf{p}_{k}, \mathbf{p}_{-k}\right) \triangleq-\log \left(\frac{1}{N_{k} m_{k}} \sum_{n \in \mathcal{N}_{k}} \sum_{\mu=1}^{\sqrt{2^{m} k} / 2} \alpha_{k, \mu} \mathrm{e}^{-\frac{p_{k, n} \gamma_{k, n}\left(\mathbf{p}_{-k, n}\right)}{\psi_{k, \mu}}}\right)$,

where $\mathbf{p}_{-k} \triangleq\left[\mathbf{p}_{1}^{\mathrm{T}}, \cdots, \mathbf{p}_{k-1}^{\mathrm{T}}, \mathbf{p}_{k+1}^{\mathrm{T}}, \cdots, \mathbf{p}_{K}^{\mathrm{T}}\right]^{\mathrm{T}}$ and $\mathbf{p}_{-k, n} \triangleq$ $\left[p_{1, n}, \cdots, p_{k-1, n}, p_{k+1, n}, \cdots, p_{K, n}\right]^{\mathrm{T}}$ collect all the PA vectors and per-subcarrier PA coefficients except those associated to user $k$, respectively, and $\alpha_{k, \mu}$ and $\psi_{k, \mu}$ are constant values related to the modulation size adopted by the $k$ th user. Their values are evaluated in [31] and reported in Table 1. It can be noted that the channel gain (5) has been explicitly expressed as a function of $\mathbf{p}_{-k, n}$.

\subsection{Problem formulation}

Exploiting the $\kappa$ ESM LPP method recalled in Section 3.1, the EGP metric, defined as the number of information bits delivered in error-free packets per unit of time, is expressed in (bit/OFDM block) as [31]

$$
\zeta_{k}\left(\boldsymbol{\varphi}_{k}, \mathbf{p}_{k}, \mathbf{p}_{-k}\right) \triangleq \breve{\zeta}_{k} r_{k} N_{k} m_{k}\left[1-\Phi_{r_{k}}\left(\bar{\gamma}_{k}\left(\boldsymbol{\varphi}_{k}, \mathbf{p}_{k}, \mathbf{p}_{-k}\right)\right)\right],
$$

with $\breve{\zeta}_{k} \triangleq U_{k}^{(\mathrm{p})} / U_{k}^{(\mathrm{i})}$, being $U_{k}^{(\mathrm{p})}$ the number of payload bits. Let us assume a given QoS per user in terms of a target EGP value $\bar{\zeta}_{k}$. The aim is to consume the lowest power as possible, while meeting such EGP QoS constraints. Besides, due to the mutual interference, the EGP of a given user is affected by the transmission power employed by the other users. Thus, assuming the lack of a central coordinating unit, the goal of the users is to reach, in a distributed way, the optimal PA $\mathbf{p}_{k}^{*} \triangleq\left[p_{k, 1}^{*}, \cdots, p_{k, N}^{*}\right]^{\mathrm{T}}$ and the optimal TM $\varphi_{k}^{*}, \forall k \in \mathcal{K}$, which satisfy the QoS constraints spending the minimum transmission power. This problem, tagged as power-efficient under guaranteed EGP OP, or PEGE for short, can thus be formalized as

$$
\begin{aligned}
& \min _{\boldsymbol{\varphi}_{k}, \mathbf{p}_{k}}\left\{u_{k}\left(\mathbf{p}_{k}\right) \triangleq \sum_{n=1}^{N} p_{k, n}\right\} \\
& \forall k \in \mathcal{K}: \begin{array}{l}
\text { s.t. } \quad \zeta_{k}\left(\boldsymbol{\varphi}_{k}, \mathbf{p}_{k}, \mathbf{p}_{-k}\right) \geq \bar{\zeta}_{k} \\
p_{k, n} \geq 0, \quad \forall n \in \mathcal{N}_{k}, \\
\boldsymbol{\varphi}_{k} \in \mathcal{D}_{r} \times \mathcal{D}_{\mathbf{m}}
\end{array} .
\end{aligned}
$$

Table 1 Values of $\kappa$ ESM coefficients for 4-, 16-, and 64-QAM

\begin{tabular}{llllll}
\hline & & \multicolumn{5}{c}{$\mu$} \\
\cline { 3 - 6 } & & 1 & 2 & 3 & 4 \\
\hline $2^{m-1} \alpha_{\mu}$ & 4-QAM & 4 & 0 & 0 & 0 \\
& 16-QAM & 24 & 8 & 0 & 0 \\
& 64-QAM & 112 & 48 & 16 & 16 \\
$\psi_{\mu}$ & 4-QAM & 2 & $1 / 2$ & $2 / 9$ & $1 / 8$ \\
& 16-QAM & 10 & $5 / 2$ & $10 / 9$ & $5 / 8$ \\
& 64-QAM & 42 & $21 / 2$ & $14 / 3$ & $21 / 8$ \\
\hline
\end{tabular}


A few remarks about (9) are now in order.

1) The OP (9), when applied to a P2P BIC-OFDM link, has not yet been taken into consideration, and so, it lacks analysis. Therefore, the above issue is first addressed in Section 4, where we derive a closed-form solution for the PA, being it useful for the subsequent case of multiple users addressed in Section 5.

2) In view of the QoS contraints, there exists a competition among the multiple active users allowed to transmit over the same band. Thus, the problem falls in the NCG framework, which is efficiently employed to study the strategic inter-user interactions [35]. Hence, the OP (9) can be formulated as a NCG, whose conditions of existence and uniqueness of the solution are analytically derived in Section 5, together with a distributed implementation of the RA algorithm.

3) The total power constraint (1) for each user is skipped in the OP (9), since its presence makes the game analysis impractical. Hence, we assume that the optimal PA, satisfying the QoS constraint, satisfies (1) as well. Nevertheless, when introducing the distributed RA algorithm in Section 5.3, such a power constraint will be restored, giving additional comments and insights on it.

\section{PEGE OP in point-to-point links}

Let us focus on the P2P communication link, that is $|\mathcal{Q}|=1$ and $|\mathcal{K}|=1$ (for this reason in the reminder of this section, the index $k$ will be neglected in the quantities of interest). Specifically, Section 4.1 formulates the strategy to select the optimal TM and PA vector solving OP (9), whereas Section 4.2 solves the PA problem in closedform.

\subsection{Problem formulation}

As for the solution of OP (9), it can be pointed out that (i) even if uniform BL is chosen for each user, the choice of the active subcarriers entails again a BL procedure, since the $n$th component of the BL $\mathbf{m}$ is $m_{n}=m$ if subcarrier $n$ is active, $m_{n}=0$ otherwise, as described in Section 3.1; (ii) the BL and PA problems cannot be jointly solved, in that the problem is NP-hard. Given the QoS constraint and the SINR vector $\gamma$, however, the OP can be efficiently yet suboptimally tackled relying on the following strategy: first, the optimal PA vector $\mathbf{p}^{*} \triangleq\left[p_{1}^{*}, \cdots, p_{N}^{*}\right]^{\mathrm{T}}$ is derived as a function of the generic TM $\varphi$, i.e., $\mathbf{p}^{*} \equiv \mathbf{p}^{*}(\varphi)$, and, then, the pair TM and PA that minimizes the power consumption while satisfying the QoS constraint is selected as solution to the OP.

PA in PEGE OP for a fixed TM. Assuming a given TM $\varphi$, the optimal PA $\mathbf{p}^{*}(\varphi)$ solving OP (9) is obtained as

$$
\begin{gathered}
\mathbf{p}^{*}(\boldsymbol{\varphi})=\underset{\mathbf{p}}{\operatorname{argmin}}\{u(\mathbf{p})\} \\
\text { s.t. } \sum_{n=1}^{N} \sum_{\mu=1}^{\sqrt{2^{m}} / 2} \alpha_{\mu}(\boldsymbol{\varphi}) \mathrm{e}^{-\frac{p_{n} \gamma_{n}}{\psi_{\mu}(\boldsymbol{\varphi})}} \leq \kappa(\boldsymbol{\varphi}) \\
p_{n} \geq 0
\end{gathered}
$$

where the first constraint in (10) corresponds to the QoS constraint of (9) expressed as a function of the PA, with, in view of (7) and (8),

$$
\kappa(\varphi) \triangleq N m \mathrm{e}^{-\gamma^{*}(\varphi)}
$$

and

$$
\gamma^{*}(\varphi) \triangleq \Phi^{-1}(1-\bar{\zeta} /(\breve{\zeta} r N m)),
$$

being constant values, both depending only on $\varphi$. From Eqs. (11)-(12), it is seen that, given the BL vector and the coding rate, the QoS constraint $\bar{\zeta}$ can be equivalently expressed as a function of the target $\operatorname{ESNR} \gamma^{*}(\varphi)$.

Nevertheless OP (10), having a linear objective function and convex constraints, be a convex OP, due to the presence of the QoS constraint it does not present a closedform solution. Therefore, solving (10) can be approached via conventional numerical methods [36], though at the price of a high computational load. Afterward, we propose an alternative approximate yet efficient method to get a closed-form solution of OP (10). Hence, the numerical computationally heavy solution will be only used as benchmark to test the accuracy of the proposed approximated closed-form solution.

TM selection in PEGE OP. Given the SINR $\gamma$ and assuming uniform $\mathrm{PA}$ across the subcarriers ${ }^{1}$, first the $\mathrm{BL}$ procedure originally proposed in [37] is applied, with the set of feasible number of bits per subcarrier being $\{0, m\}$. As a result, we get $\left|\mathcal{D}_{m}\right| \cdot N$ possible BL vectors $\left\{\overline{\mathbf{m}}^{(n, m)}\right\}$, $n=1, \cdots, N$, with $m \in \mathcal{D}_{m}$. As a matter of fact, $\overline{\mathbf{m}}^{(n, m)}$ denotes the best bit allocation vector with $n$ active subcarriers and $m$ bits per active subcarrier. Let us denote with $\mathcal{D}_{\overline{\mathbf{m}}} \subset \mathcal{D}_{\mathbf{m}}$ the set collecting such vectors. Then, if the PA $\mathbf{p}^{*}(\varphi)$ are available for the TMs $\varphi \in \mathcal{D}_{r} \times \mathcal{D}_{\overline{\mathbf{m}}}$, the OP (9) is solved by searching the best TM $\varphi^{*}$ that both minimizes the power consumption and satisfies the QoS constraint. Formally,

$$
\begin{array}{cc}
\varphi^{*}=\underset{\varphi}{\operatorname{argmin}}\left\{u\left(\mathbf{p}^{*}(\varphi)\right)\right\} \\
\text { s.t. } \quad \zeta\left(\boldsymbol{\varphi}, \mathbf{p}^{*}(\boldsymbol{\varphi})\right) \geq \bar{\zeta}, \\
& \boldsymbol{\varphi} \in \mathcal{D}_{r} \times \mathcal{D}_{\overline{\mathbf{m}} .}
\end{array}
$$

The OP (13) can thus be solved by means of the pseudocode outlined in Table 2. The key points of the proposed procedure are as follows. (i) For every $m \in \mathcal{D}_{m}$, the set $\mathcal{D}_{\overline{\mathbf{m}}}$ is efficiently found exploiting the BL strategy [37], which is based on a greedy algorithm with complexity $\mathcal{O}(N \log N)$. (ii) The best TM is obtained carrying out 
Table 2 TM selection algorithm

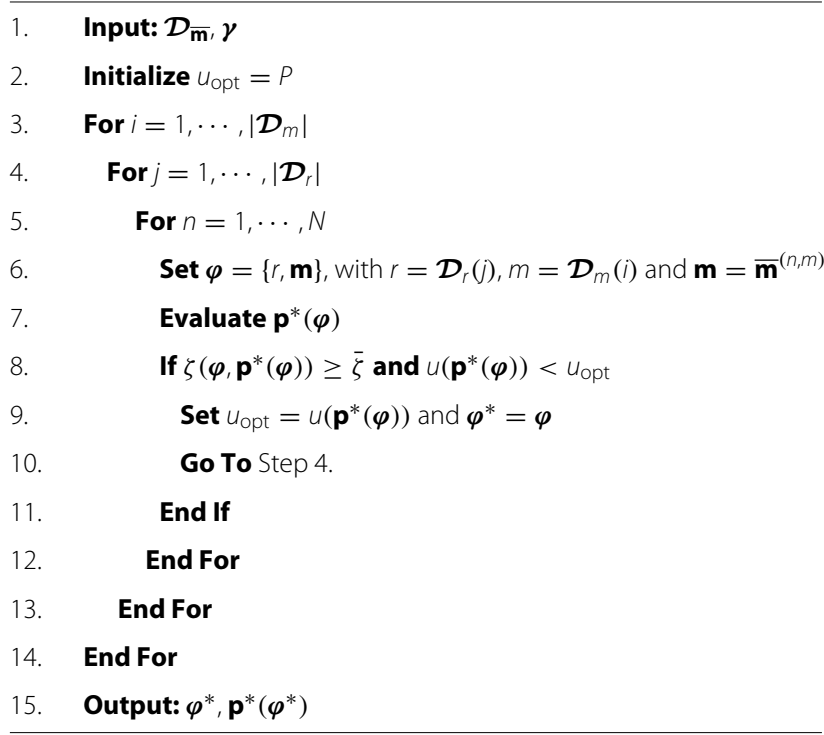

an exhaustive search over the possible TMs. The latter stands for the worst case condition, in that, for a given pair $\{r, m\}$, the search is halted as soon as a feasible TM is found (step 10 of Table 2), because any other TM with the same pair $\{r, m\}$ would require a larger amount of transmitted power. Indeed, for a given $m, \overline{\mathbf{m}}^{(n+1, m)}$ would have the same active subcarriers as $\overline{\mathbf{m}}^{(n, m)}$ plus an additional active subcarrier chosen among the remaining $N-n$ ones. As a consequence, the power consumption of the former case would be greater than that of the latter one. (iii) In order to maintain an affordable complexity of the overall algorithm, a closed-form expression of the PA can be derived, as illustrated in the next section.

\subsection{Closed-form solution for the PA problem}

Let us now point out that the summation over $\mu$ in the QoS constraint prevents to easily obtain a closed-form solution to the PA problem. Thus, the idea we pursue is to approximate the QoS expression in (10) by introducing a scalar $\beta$ such that a possible closed-form PA solution matches the optimal one earned by solving (10) via a numerical (computationally heavy) method.

Proposition 1 Approximating the left-hand side of the QoS constraint in (10) with $\beta \sum_{n=1}^{N} \mathrm{e}^{-\frac{p_{n}}{\rho_{n}}}$, with $\rho_{n} \triangleq \delta / \gamma_{n}$, $\beta \in \mathbb{R}^{+}$and $\rho \in \mathbb{R}^{+}$, the OP (10) turns into

$$
\begin{gathered}
\min _{\mathbf{p}} u(\mathbf{p}) \\
\text { s.t. } \beta \sum_{n=1}^{N} \mathrm{e}^{-\frac{p_{n}}{\rho_{n}}} \leq \kappa \\
p_{n} \geq 0 .
\end{gathered}
$$

So doing, the nth component of the solution $\mathbf{p}^{\star}$ to (14) can be written in closed-form as the water-filling-like solution [20]

$$
p_{n}^{\star}=\rho_{n}\left[\log \Theta-\log \frac{\rho_{n}}{\beta}\right]^{+}, \quad \forall n \in \mathcal{N},
$$

where $\Theta$ is such that $\mathbf{p}^{\star}$ satisfies the QoS constraint in (14) with equality and represents the "water-level."

Proof The proof is given in Appendix A.

Concerning the values of $\beta$ and $\delta$, by looking at the values assumed by the coefficients $\alpha_{\mu}$ and $\psi_{\mu}$ for a given modulation order (Table 1), it follows that the left-hand side of the QoS constraint in (10) can be lower and upper bounded as

$$
\beta^{(\mathrm{l})} \sum_{n=1}^{N} \mathrm{e}^{-\frac{p_{n} \gamma_{n}}{\delta^{(\mathrm{l})}}} \leq \sum_{n=1}^{N} \sum_{\mu=1}^{\sqrt{2^{m}} / 2} \alpha_{\mu} \mathrm{e}^{-\frac{p_{n} \gamma_{n}}{\psi_{\mu}}} \leq \beta^{(\mathrm{u})} \sum_{n=1}^{N} \mathrm{e}^{-\frac{p_{n} \gamma_{n}}{\delta(\mathrm{u})}},
$$

where $\delta^{(\mathrm{l})} \triangleq \psi_{1}, \beta^{(\mathrm{l})} \triangleq \alpha_{1}, \delta^{(\mathrm{u})} \triangleq \psi_{1}$ and $\beta^{(\mathrm{u})} \triangleq \sum_{\mu=1}^{\sqrt{2^{m}} / 2} \alpha_{\mu}$. The strict equality holds for $m=2$, since in this case the sum over $\mu$ reduces exactly to only one term.

Denoting with $\mathbf{p}^{(\mathrm{l})}$ and $\mathbf{p}^{(\mathrm{u})}$ the optimal solutions (15) when the pair $\{\beta, \delta\}$ is set in Eqs. (14)-(15) to $\left\{\beta^{(\mathrm{l})}, \delta^{(\mathrm{l})}\right\}$ and $\left\{\beta^{(\mathrm{u})}, \delta^{(\mathrm{u})}\right\}$, respectively, the drawback is that the solution associated to the lower bound (LB) tends to underestimate the QoS constraint and, thus, to allocate less power compared to the optimal solution. On the other hand, the solution associated to the upper bound (UB) overestimates the QoS constraint and, thus, the performance in term of EGP are always satisfied, although at the price of spending more power than the strictly required one. Hence, the idea is to find the optimal pair $\left\{\beta^{(0)}, \delta^{(o)}\right\}$ that minimizes the mean square error (MSE) between the exact expression of the QoS constraint in (10) and the proposed expression of the QoS constraint in (14), for every modulation order.

The results depicted in Figs. 3 and 4 for the BIC-OFDM link with the setup of Table 3 , employing $(r, m)=(1 / 3,4)$ and $(r, m)=(2 / 5,6)$, respectively, show the minimum transmitted power versus the symbol energy-to-noise power spectral density ratio $E_{s} / N_{0}$, assuming a target EGP QoS $\bar{\zeta}=0.31 \mathrm{bit} / \mathrm{s} / \mathrm{Hz}$. In line with (16), the PAs obtained by employing $\left\{\beta^{(\mathrm{l})}, \delta^{(\mathrm{l})}\right\}$ and $\left\{\beta^{(\mathrm{u})}, \delta^{(\mathrm{u})}\right\}$ lead to values of the objective function lower and higher, respectively, than the optimal ones (in accordance with the fact that they are a lower and upper bound of the QoS constraint). Conversely, the PA performance obtained with $\left\{\beta^{(\mathrm{o})}, \delta^{(\mathrm{o})}\right\}$ closely matches that obtained via numerical 


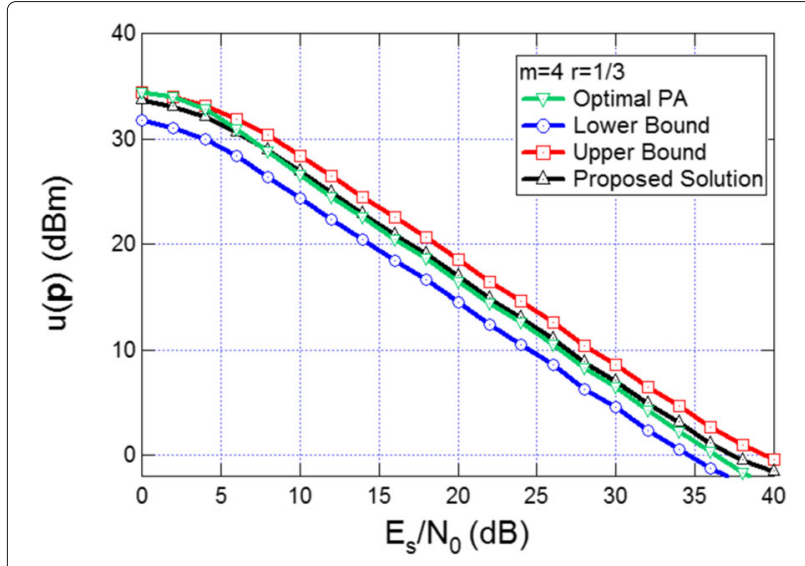

Fig. 3 Performance comparison of the proposed PA algorithms for $(r, m)=(1 / 3,4)$

methods, while exploiting an efficient closed-form expression. The optimal values of $\left\{\beta^{(0)}, \delta^{(0)}\right\}$ that, for every modulation order, minimize the MSE betweem the optimal and the proposed expression of the QoS constraint have been computed for $E_{s} / N_{0} \in[-6,30] \mathrm{dB}$ and can be found in Table 4. In the case of multiple users access, such an approximation will be utilized for evaluating the QoS constraint in OP (9).

\section{PEGE OP over multi-access interference links}

We assume now that there not exist any centralized unit, and so, users coordinate among themselves in a distributed manner in order to reach a stable RA configuration. The goal is to design a distributed algorithm so that each user minimizes its power consumption, while satisfying its QoS constraint and accounting for the interference caused by the other users exploiting the same frequencies.

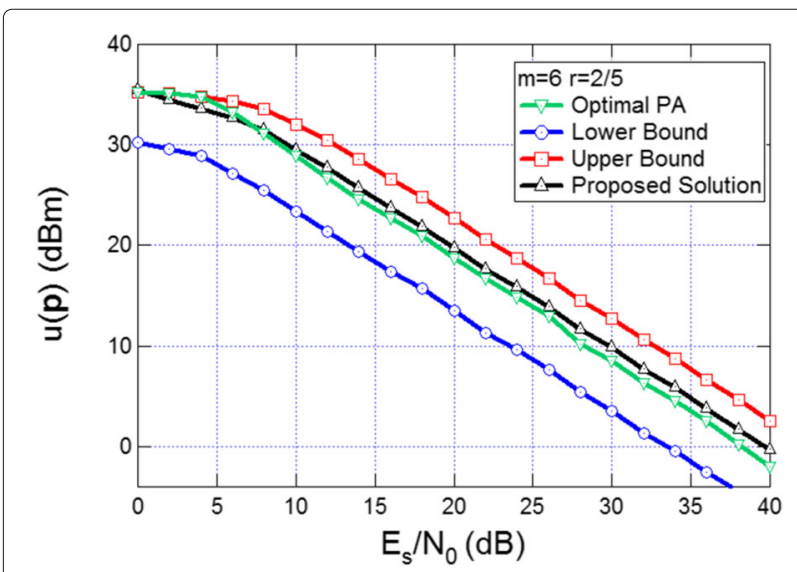

Fig. 4 Performance comparison of the proposed PA algorithms for $(r, m)=(2 / 5,6)$

\subsection{Game formulation}

In order to derive a distributed algorithm solving OP (9), let us consider for the time being a given TM $\varphi_{k}$ per user. Then, resorting to the QoS constraint approximation adopted in (14), the PEGE OP (9) can be reformulated as

$$
\begin{gathered}
\min _{\mathbf{p}_{k}} u_{k}\left(\mathbf{p}_{k}\right) \\
\forall k \in \mathcal{K} \text { : s.t. } \beta_{k} \sum_{n \in \mathcal{N}_{k}} \mathrm{e}^{-\frac{p_{k, n}}{\rho_{k, n}\left(\mathbf{p}_{-k, n}\right)}} \leq \kappa_{k}, \\
p_{k, n} \geq 0,
\end{gathered}
$$

where $\kappa_{k}$ is evaluated $\forall k \in \mathcal{K}$ via (11)-(12),

$$
\rho_{k, n}\left(\mathbf{p}_{-k, n}\right) \triangleq \frac{\delta_{k}}{\gamma_{k, n}\left(\mathbf{p}_{-k, n}\right)},
$$

and $\beta_{k}$ and $\delta_{k}$ assume values reported in Table 4. OP (17) can be solved by modeling it as a NCG [35]. Such a framework offers a powerful analytical tool that describes how rational entities interact and make appropriate choices so that they can find their own maximum utility.

Accordingly, we introduce the game $\mathcal{G} \triangleq\{\mathcal{K}, \mathcal{P}, \mathcal{U}\}$, described as follows:

1) $\mathcal{K} \triangleq \mathcal{K}_{1} \times \cdots \times \mathcal{K}_{Q}$ is the overall set of users, i.e., players.

2) $\mathcal{P} \triangleq \mathcal{P}_{1} \times \cdots \times \mathcal{P}_{K}$ is the set strategies, where the strategy of user $k$ is its feasible PA set, defined as

$$
\begin{aligned}
\mathcal{P}_{k} \triangleq\left\{p_{k, n} \mid g_{k}\left(\mathbf{p}_{k}, \mathbf{p}_{-k}\right)\right. & \leq 0, \quad p_{k, n} \\
& \left.\geq 0, \forall n \in \mathcal{N}_{k}\right\}, \quad \forall k \in \mathcal{K},
\end{aligned}
$$

with $g_{k}\left(\mathbf{p}_{k}, \mathbf{p}_{-k}\right) \triangleq \sum_{n \in \mathcal{N}_{k}} \beta_{k} \mathrm{e}^{-\frac{p_{k, n}}{\rho_{k, n}\left(\mathbf{p}_{-k, n}\right)}}-\kappa_{k}$.

3) $\mathcal{U} \triangleq\left\{u_{1}, \cdots, u_{K}\right\}$ is the set collecting the utility functions defined in (9).

By looking at the elements identifying $\mathcal{G}$, it worth pointing out that the QoS constraints introduce an interdependency among the strategies of the players, i.e., $\mathcal{P}_{k}=$ $\mathcal{P}_{k}\left(\mathbf{p}_{k}, \mathbf{p}_{-k}\right)$. In other words, the set of strategies of the generic player $k$ depends on the other players' strategies. The solution of game (17) is thus investigated in terms of GNE, which corresponds to the case where no player can decrease his objective function by changing unilaterally its strategy to any other feasible one [32]. Equivalently, a GNE of the game is reached when every user, given the strategy profile of the others, does not get any power decrease by unilaterally changing its own strategy, still keeping the QoS constraint satisfied. Formally, the vector $\mathbf{p}^{*} \triangleq\left[\mathbf{p}_{1}^{* \mathrm{~T}}, \cdots, \mathbf{p}_{K}^{* \mathrm{~T}}\right]^{\mathrm{T}} \in \mathcal{P}$, which represents now the optimum power allocation for all the users, is a GNE [35] for game $\mathcal{G}$ if

$$
u_{k}\left(\mathbf{p}_{k}^{*}\right) \leq u_{k}\left(\mathbf{p}_{k}^{\prime}\right), \forall \mathbf{p}_{k}^{\prime} \in \mathcal{P}_{k}\left(\mathbf{p}_{k}^{\prime}, \mathbf{p}_{-k}^{*}\right), \forall k \in \mathcal{K} .
$$


Table 3 BIC-OFDM link setup

\begin{tabular}{ll}
\hline Parameter & Value/description \\
\hline Payload length $N_{P}^{(p)}$ & 1024 bits \\
RLC-PDU length $N^{(i)}$ & 1056 bits \\
N. of active subcarriers $N$ & 1320 \\
FFT size & 2048 \\
RF bandwidth/subcarrier spacing & $20 \mathrm{MHz} / 15.152 \mathrm{kHz}$ \\
Coding scheme & Parallel concatenated convolutional coding (PCCC) turbo codes $\mathcal{D}_{r}=\left\{\frac{1}{3}, \frac{2}{5}, \frac{1}{2}, \frac{4}{7}\right\}$ \\
Modulation & $4-, 16-, 64-\mathrm{QAM}$ \\
Available power $P$ & $33 \mathrm{dBm}$ \\
Channel model & 6 -tap power profile of ITU-Pedestrian B
\end{tabular}

In order to study the game equilibrium, let us point out the following remark.

Remark 1 For a given strategy $\mathbf{p}_{-k}$ of the other players, the solution of (17) corresponds to the solution found for the P2P link (15). Thus, in this case the solution is unique and given, capitalizing on the approximate yet accurate closed-form one, by

$$
\mathbf{p}_{k}^{*}=\mathrm{BR}\left(\mathbf{p}_{-k}\right), \quad \forall k \in \mathcal{K},
$$

where the nth component of the best response (BR) operator is defined as

$$
\begin{gathered}
{\left[\mathrm{BR}\left(\mathbf{p}_{-k}\right)\right]_{n} \triangleq \rho_{k, n}\left(\mathbf{p}_{-k, n}\right)\left[\log \Theta_{k}^{*}-\log \frac{\rho_{k, n}\left(\mathbf{p}_{-k, n}\right)}{\beta_{k}}\right]^{+},} \\
\forall n \in \mathcal{N}_{k}, \forall k \in \mathcal{K},
\end{gathered}
$$

and $\Theta_{k}^{*}$ is such that the optimal PA $\mathbf{p}_{k}^{*}$ satisfies the QoS constraints with equality.

Thus, according to the definition of GNE and in view of Remark 1, the GNE of the game must satisfy the following condition.

Proposition 2 If problem (17) is feasible, i.e., if there exist a $P A$ vector $\mathbf{p}^{(\mathrm{f})} \geq \mathbf{0}$ such that QoS constraints are met with equality, then there exists at least one $P A \mathbf{p}^{*}$ which is a GNE equilibrium of the game. Moreover, the GNE has to satisfy the so-called best response solution for each user, by solving the fixed-point system of equations

$$
\mathbf{p}_{k}^{*}=\mathrm{BR}\left(\mathbf{p}_{-k}^{*}\right), \quad \forall k \in \mathcal{K},
$$

with the operator BR defined as in (22).

Table 4 Optimal values of $\left\{\beta^{(0)}, \delta^{(0)}\right\}$ for every modulation order

\begin{tabular}{llll}
\hline & 4-QAM & 16-QAM & 64-QAM \\
\hline$\beta^{(0)}$ & 2 & 3.716 & 8.244 \\
$\delta^{(0)}$ & 2 & 5.407 & 25.9134 \\
\hline
\end{tabular}

We remark here that the fixed-point system of Eq. (23) may lead to more than one solution, or the solution of game (17) may not exist, since there may not exist a $\mathbf{p}^{*}$ that satisfies all the QoS constraints of all the users at the same time. Thus, before going into details of how to solve (17), in the next section we focus on the feasibility conditions of OP (17), as well as on the existence and uniqueness of the GNE of the game describing OP (17).

\subsection{GNE analysis}

In order to derive sufficient conditions for the feasibility of OP (17) and the existence of at least a solution for the associated game, given the vector of required EGP values, or equivalently, the corresponding vector of optimal ESNR $\boldsymbol{\gamma}^{*} \triangleq\left[\gamma_{1}^{*}, \cdots, \gamma_{K}^{*}\right]^{\mathrm{T}}$, with $\gamma_{k}^{*}$ defined in (12), let us introduce the following definitions:

$$
\mathbf{t}_{n}\left(\boldsymbol{\gamma}^{*}\right) \triangleq\left[\tilde{\gamma}_{1}^{*} \sigma_{1, n}^{2}, \cdots, \tilde{\gamma}_{K}^{*} \sigma_{K, n}^{2}\right]^{\mathrm{T}},
$$

and

$$
\mathbf{Z}_{n}\left(\gamma^{*}\right) \triangleq\left[\begin{array}{cccc}
\left|\bar{h}_{1, \phi(1), n}\right|^{2} & -\tilde{\gamma}_{1}^{*}\left|h_{2, \phi(1), n}\right|^{2} & \cdots & -\tilde{\gamma}_{1}^{*}\left|h_{K, \phi(1), n}\right|^{2} \\
-\tilde{\gamma}_{2}^{*}\left|h_{1, \phi(2), n}\right|^{2} & \left|\bar{h}_{2, \phi(2), n}\right|^{2} & \cdots & -\tilde{\gamma}_{2}^{*}\left|h_{K, \phi(2), n}\right|^{2} \\
\vdots & \vdots & \ddots & \vdots \\
-\tilde{\gamma}_{K}^{*}\left|h_{1, \phi(K), n}\right|^{2} & -\tilde{\gamma}_{K}^{*}\left|h_{2, \phi(K), n}\right|^{2} & \cdots & \left|\bar{h}_{K, \phi(K), n}\right|^{2}
\end{array}\right],
$$

where $\bar{h}_{k, \phi(k), n} \triangleq h_{k, \phi(k), n} / \delta_{k}$ and $\tilde{\gamma}_{k}^{*} \triangleq \gamma_{k}^{*}+\log \beta_{k}$, being $\delta_{k}$ and $\beta_{k}$ constant values defined in Table 4.

Proposition 3 Given $\boldsymbol{\gamma}^{*}$, a sufficient condition for the feasibility of (17) is that $\mathbf{Z}_{n}\left(\boldsymbol{\gamma}^{*}\right)$, defined in (25), is a P-matrix.

Proof See Appendix B.

Capitalizing on the feasibility condition derived above, the existence of at least one GNE is guaranteed, as stated in the following proposition. 
Proposition 4 Given $\boldsymbol{\gamma}^{*}$ and assuming that problem (17) is feasible, then there exists at least a bounded GNE for game (17). Furthermore, any GNE $\mathbf{p}^{*}$, which can be equivalently expressed as $\mathbf{p}^{*} \triangleq\left[\mathbf{q}_{1}^{* \mathrm{~T}}, \cdots, \mathbf{q}_{N}^{* \mathrm{~T}}\right]^{\mathrm{T}}$, with $\mathbf{q}_{n}^{*} \triangleq$ $\left[p_{1, n}^{*}, \cdots, p_{K, n}^{*}\right]^{\mathrm{T}}, 1 \leq n \leq N$, is such that

$$
\mathbf{q}_{n}^{*} \leq \overline{\mathbf{q}}_{n}=\left[\mathbf{Z}_{n}\left(\boldsymbol{\gamma}^{*}\right)\right]^{-1} \cdot \mathbf{t}_{n}\left(\boldsymbol{\gamma}^{*}\right), \quad \forall n,
$$

where $\overline{\mathbf{q}}_{n} \triangleq\left[\bar{p}_{1, n}, \cdots, \bar{p}_{K, n}\right]^{\mathrm{T}}$.

Proof See Appendix C.

In order to better understand the physical meaning of the existence condition, let us first express the channel coefficient as a function of the path loss (PL) between the relevant transmitter and receiver pair, i.e., $\left|\tilde{h}_{j, \phi(k), n}\right|^{2} \triangleq$ $\left|h_{j, \phi(k), n}\right|^{2} L_{j, \phi(k)}$, where $L_{j, \phi(k)}$ is the path loss between between user $j$ and BS $\phi(k)$ and $\tilde{h}_{j, \phi(k), n} \in \mathcal{C N}(0,1)$. Since a sufficient condition for the matrices $\left\{\mathbf{Z}_{n}\right\}$ to be $P$-matrices is that they satisfy the diagonal dominance condition [38], then the following corollary holds.

Corollary 1 Sufficient conditions for the matrices $\left\{\mathbf{Z}_{n}\right\}$ to be P-matrices, and hence for the solution set of game (17) to be non-empty, are

$$
\sum_{\substack{j=1 \\ j \neq k}}^{K} \frac{\left|\tilde{h}_{j, \phi(k), n}\right|^{2}}{\left|\tilde{h}_{k, \phi(k), n}\right|^{2}} \frac{L_{k, \phi(k)}}{L_{j, \phi(k)}}<\frac{\delta_{k}}{\tilde{\gamma}_{k}^{*}} \quad \forall n \in \mathcal{N}_{k}, \forall k \in \mathcal{K},
$$

which can be derived by exploiting the definition of diagonal dominance of a matrix.

Thus, the above condition states that as long as the ratio between the interfering channel and the direct one, i.e, the direct channel between the transmitter and the receiver, is lower than a certain threshold, or, in other words, the interference is small enough, then a solution for game (17) exists. The threshold depends on the EGP constraints, expressed in terms of optimal ESNR, along with the modulation order adopted, via the coefficients $\delta_{k}$ and $\beta_{k}$ (the latter contained in $\tilde{\gamma}_{k}^{*}$ ). Thus, the more distant the competing transmitters, the lower the interference and, thus, the higher the probability of having a non-empty solution set $[21,23]$. Further, it is worth noting that the condition formulated in (27) is in agreement with that derived in [23] with Gaussian signaling. In this latter case, indeed, $\delta_{k}=1$ and $\beta_{k}=1$ as well, thus implying $\tilde{\gamma}_{k}^{*}=\gamma_{k}^{*}$, and $\gamma_{k}^{*}=\mathrm{e}^{R_{k}^{*}}-1$, where $R_{k}^{*}$ is the desired rate.

Finally, we focus on the sufficient conditions for the uniqueness of the GNE. This analysis, however, is made difficult by the presence of the QoS constraints, that introduces an interdependency among the strategy sets of the users. Thus, partly inspired by [23], the derivation of the uniqueness conditions is carried out as follows: first, a change of variable is done in order to obtain an equivalent formulation of the problem as a variational inequality (VI) problem; then, the conditions of uniqueness of the solution for the original problem are derived.

Proposition 5 Given $\gamma^{*}$ and under the assumption that the problem is feasible, a sufficient condition for the uniqueness of the GNE for game (17) is that the matrix $\mathbf{V}\left(\gamma^{*}\right)$, defined in (66), is a P-matrix.

Proof See Appendix D.

As expected, also the uniqueness condition depends on the target ESNR $\gamma^{*}$ and on the ratio among the direct and interference channels, which appears on the off-diagonal elements of $\mathbf{V}\left(\boldsymbol{\gamma}^{*}\right)$ through the parameter $\chi_{j, k}^{(\max )}$ defined in (64). Thus, although condition of Proposition 5 is less easy to check than the one corresponding to the existence of the GNE, from (66) it can be inferred that whenever the ratio between each direct and interference channel is small, matrix $\mathbf{V}\left(\gamma^{*}\right)$ is likely to satisfy the diagonal dominance property and thus to be a $P$-matrix, ensuring the uniqueness of the GNE.

\subsection{Distributed algorithm}

Since we are dealing with a decentralized implementation, where no signaling among different BSs is allowed, our aim is to derive a totally distributed algorithm. In fact, this allows every user to independently optimize its own PA, as well as TM, according to the perceived SINR, which entails the interference caused by the other users. Recalling Proposition 1, a natural scheme is a distributed and iterative algorithm based on the BR. In particular, at each iteration $j$, considering the generic user $k \in \mathcal{K}$ and the PA $\mathbf{p}_{-k}^{(j-1)}$ chosen by the other users at the previous iteration, the PA vector $\mathbf{p}_{k}^{(j)}$ is evaluated, as function of the selected TM $\varphi_{k}$, according to (22), $\forall k \in \mathcal{K}$. This means that, to perform the update, the only information required by every user $k$ is the SINR vector $\boldsymbol{\gamma}_{k}\left(\mathbf{p}_{-k}^{(j-1)}\right)$ in (5). This is measured at the serving BS and sent with a modest feedback rate requirement on the return channel. Then indeed, since every user is able to evaluate, at every iteration $j$, the best $\operatorname{PA} \mathbf{p}_{k}^{(j)}\left(\boldsymbol{\varphi}_{k}\right)$ as a function of the TM $\varphi_{k}$ and SINR, the selection of the best pair $\left\{\boldsymbol{\varphi}_{k}^{(j)}, \mathbf{p}_{k}^{(j)}\left(\boldsymbol{\varphi}_{k}^{(j)}\right)\right\}$ can be carried out in the same manner of Section 4.1 according to the algorithm in Table 2. This procedure is iterated for all the users until convergence is reached, and it is summarized in Table 5, where $\epsilon_{p}$ denotes the required solution accuracy, $N_{\text {it }}$ is 
Table 5 Distributed RA algorithm

\begin{tabular}{ll}
\hline 1. & Input: $\mathbf{p}_{k}^{(0)}, \boldsymbol{\varphi}_{k}^{(0)} \forall k \in \mathcal{K}$. \\
2. & Initialize: $j=0, \epsilon_{p}$. \\
3. & Do \\
4. & For $k=1, \cdots, k$ \\
5. & Evaluate $\mathcal{D}_{\mathbf{m}}^{\left(k_{j}\right)}$ \\
6. & Evaluate $\mathbf{p}_{k}^{(j+1)}$ and $\boldsymbol{\varphi}_{k}^{(j+1)}$ applying Algorithm in Table 2 with \\
& $\quad$ Input $\boldsymbol{\mathcal { D }}_{\mathbf{m}}^{\left(k_{j}\right)}$ and $\boldsymbol{\gamma}_{k}\left(\mathbf{p}_{-k}^{(j)}\right)$ and evaluating the PA vector \\
7. & End For \\
8. & Set $j \leftarrow j+1 ;$ \\
9. & Until $\left\|\mathbf{p}^{(j)}-\mathbf{p}^{(j-1)}\right\| \leq \epsilon_{p}$ or $j=N_{\text {it }}$ \\
10. & Output: $\boldsymbol{\varphi}_{k}^{*}=\boldsymbol{\varphi}_{k}^{(j)}, \mathbf{p}_{k}^{*}=\mathbf{p}_{k}^{(j)}, \forall k \in \mathcal{K}$. \\
\hline
\end{tabular}

the maximum number of iterations, $\mathbf{p}_{k}^{(0)}$ and $\varphi_{k}^{(0)}$ are an initial feasible PA vector and TM, respectively, and $\mathcal{D}_{\overline{\mathbf{m}}}^{(k, j)}$ denotes the set of best BL vectors for user $k$ at iteration $j$, evaluated according to the perceived interference $\gamma_{k}\left(\mathbf{p}_{-k}^{(j)}\right), \forall k \in \mathcal{K}$.

Remark 2 The proposed best response-based algorithm converges under the same conditions for which the GNE is unique (stated in Proposition 5), which in turn requires the condition stated in Proposition 3 in order to have a nonempty solution set. In fact the condition of convergence can be demonstrated with the same approach used to derive the uniqueness condition, simply replacing the two solutions of the GNE with two PA vectors produced by the algorithm at two consecutive iterations.

Remark 3 In the formulation of game (17), we did not consider the total power constraint per user (1). Even if this constraint is intrinsically present in wireless devices, since their power cannot grow infinitely, it makes the equilibrium analysis more involved. Then, we did not take into account this constraint in the theoretical analysis, leaving it for future research. Anyway, the following observations can be done. First, algorithm in Table 5 (as well as that for the P2P case) can easily account for constraint (1) as follows: for all $k \in \mathcal{K}$, in the optimal PA expression (22), based on the water-filling like operator, the "water-level" must now be computed as $\Lambda_{k}=\min \left\{\log \Theta_{k}^{*}, \log \tilde{\Theta}_{k}^{*}\right\}$, where $\tilde{\Theta}_{k}^{*}$ is the "water-level" that, put into the water-filling expression, returns the PA vector $\tilde{\mathbf{p}}_{k}$ that maximizes the EGP $\zeta_{k}\left(\mathbf{p}_{k}\right)$ meeting with equality the total PA constraint (1) [31]. Poorly speaking, for a given strategy $\mathbf{p}_{-k}$, the best response for user $k$ is the $P A$ vector that meets with equality the QoS constraint if the required total PA is not greater than the maximum one, otherwise, all the power is allocated returning the highest possible value of EGP. Finally, as shown in the simulation results, practical values of the
$P A$ never reach the maximum power limit, validating the theoretical results on the equilibrium analysis done.

\subsection{Relationship between GNE and PO solution}

In this section, we give some insights on the relation between the GNE and the PO solution, which represents the achievable performance upper bound. Specifically, the PO solution consists in solving a multi-objective OP, where the objective function is the sum of the utility functions of every user, i.e., $u_{\mathrm{PO}}(\mathbf{p})=\sum_{k=1}^{K} u_{k}\left(\mathbf{p}_{k}\right)$, with $\mathbf{p} \triangleq\left[\mathbf{p}_{1}^{\mathrm{T}}, \cdots, \mathbf{p}_{K}^{\mathrm{T}}\right]^{\mathrm{T}}$, and where the set of constraints is the same than that in (17). Let us refer to this problem as POOP. Besides being a non-convex OP, whose solution may be very hard to find, the PO-OP requires that every user, or a centralized entity, knows, among the others, every channel coefficient $h_{j, s, n}$ between any user and base station in the network. Obviously, this is unlikely to be feasible in the considered HetNet. However, in order to shed light on the relationship between the PO solution and the GNE, corresponding to the PA solution (23) based on the best response, we consider here a two-user two-subcarrier case $(K=2, N=2)$, where user 1 is in cell 1 and user 2 is in cell 2, i.e., $\phi(1)=1$ and $\phi(2)=2$. The simulation setup is as follows: $h_{1,1,1}=1.821, h_{1,1,2}=0.329, h_{1,2,1}=0.104$, $h_{1,2,2}=0.221, h_{2,2,1}=0.821, h_{2,2,2}=2.629, h_{2,1,1}=$ $0.319, h_{2,1,2}=0.097, \sigma_{w k, 1} / P_{k}=0.01, \forall n \in \mathcal{N}_{k}, \forall k \in \mathcal{K}$, $\left(m_{k}, r_{k}\right)=(2,1 / 3)$, and $\forall k \in \mathcal{K}$. The PO solution is found solving the PO-OP with the Matlab Global Optimization Toolbox. Finally, we consider the target EGP of user 2 to be $\bar{\zeta}_{2}=0.2(\mathrm{~b} / \mathrm{s} / \mathrm{Hz})$, whereas we let $\bar{\zeta}_{1}$ vary in the set $[0.2,1]$ (b/s/Hz). When $\bar{\zeta}_{1}=\bar{\zeta}_{2}$, only one GNE solution exists and is close to the PO one. Indeed, the ratio $\varsigma^{(\mathrm{GNE}-\mathrm{PO})}$ between the total power obtained with the GNE and PO solution, respectively, is about 0.77 . When $\bar{\zeta}_{1}=1(\mathrm{~b} / \mathrm{s} / \mathrm{Hz})$, more power needs to be allocated by user 1 , yielding a higher cochannel interference. In this case indeed 2 GNE solutions arise and $\varsigma^{(\mathrm{GNE}-\mathrm{PO})}$ drops down to about 0.56 in the worst case. From these observations, we can conclude that, as could be expected, the GNE is more efficient, i.e., closer to the $\mathrm{PO}$, when the interference in the network is lower, whereas the more severe the interference, the less efficient the GNE solution. Nevertheless, we remark that the latter allows for a distributed solution suitable for the scenario under investigation.

\section{Simulation results}

In this section, the effectiveness of the proposed distributed RA algorithm is tested. The HetNet scenario taken into consideration for the simulations is composed of one MC and $Q=4$ SCs, with $K_{0}=6$ users connected to the MBS (coverage area of radius $R_{\mathrm{MBS}}=200 \mathrm{~m}$ ) and $K_{q}=3, q=1, \cdots, Q$ users connected (coverage area of radius $R_{\mathrm{SBS}}=20 \mathrm{~m}$ ) to each SBS. Figure 5 shows a snapshot of the HetNet, whereas the packet-based BIC-OFDM 


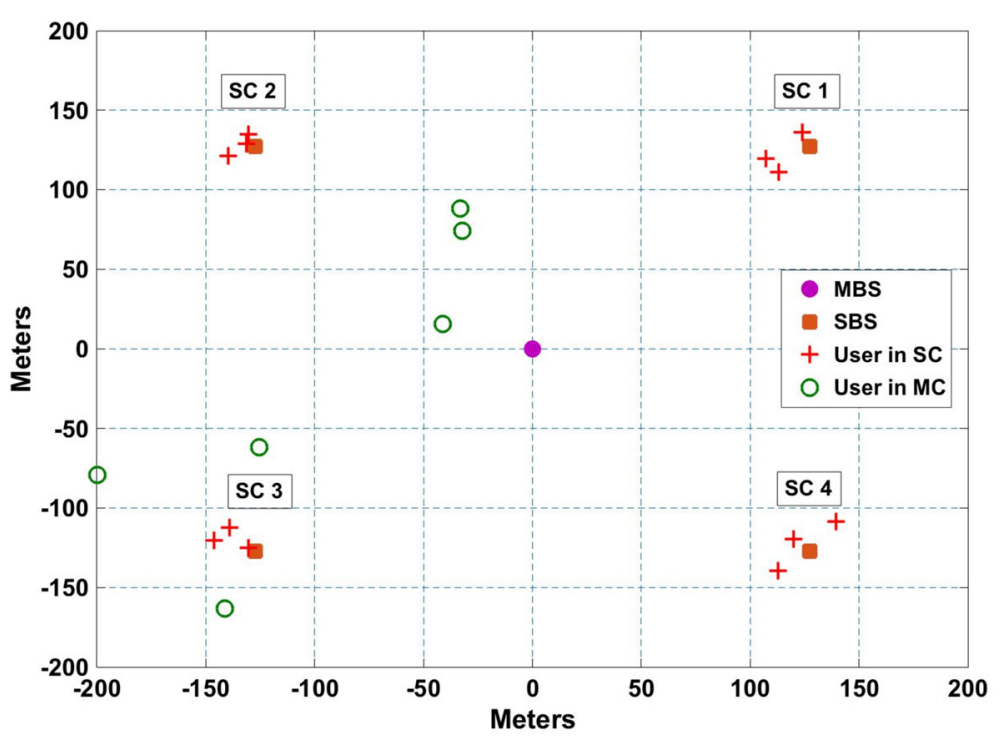

Fig. 5 HetNet simulated scenario

transmission system is summarized in Table 3. Finally, the path loss models for the $\mathrm{SC}$ and $\mathrm{MC}$ are those proposed in [39].

The target EGP QoS level $\bar{\zeta}$ is compared in Fig. 6 against the actual GP (AGP) obtained by applying the proposed distributed RA algorithm, for six users randomly chosen within MC, SC 1, SC 2, and SC 3. The AGP for the generic $k$ th user is defined as

$$
A G P_{k} \triangleq \frac{1}{N_{\mathrm{a}}} \sum_{\ell=1}^{N_{\mathrm{a}}} \frac{U_{k}^{(\mathrm{p})} \vartheta(\ell)}{T_{k}(\ell)} \quad(\mathrm{bit} / \mathrm{s} / \mathrm{Hz}),
$$

where $\vartheta(\ell)$ equals 1 if the $\ell$ th packet is correctly decoded and 0 when it is discarded, $T_{k}(\ell)$ is the transmission interval of the $\ell$ th packet, $N_{\mathrm{a}}$ is the number of trasmitted packets, set to 1000 , each experiencing independent

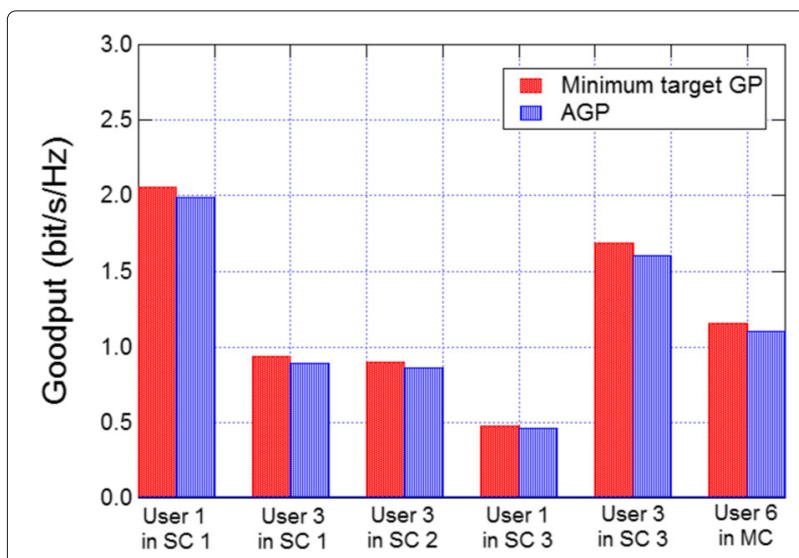

Fig. 6 Comparison between the minimum EGP QoS level and the relevant AGP for a few users selected in $\mathrm{MC}$ and SCS channel realizations and with the position of users randomly placed within their cells. In view of the effectiveness of the GP prediction based on the $\kappa \mathrm{ESM}$ model recalled in Section 3.1, it is apparent that the AGP performance meets for all the considered users, taken as example, the minimum target EGP. It can be noted that the same result holds for all the other users as well.

Figure 7 shows the total transmitted power and the EGP per user as a function of the number of the iterations of the distributed RA algorithm, obtained for a given channel realization and network geometry. As can be seen, the distributed RA algorithm guarantees fast convergence to the equilibrium point for all users of the HetNet, thus exhibiting a considerable robustness against the ICI effects.

Furthermore, in order to shed light about the convenience of employing as more SCs as possible in the

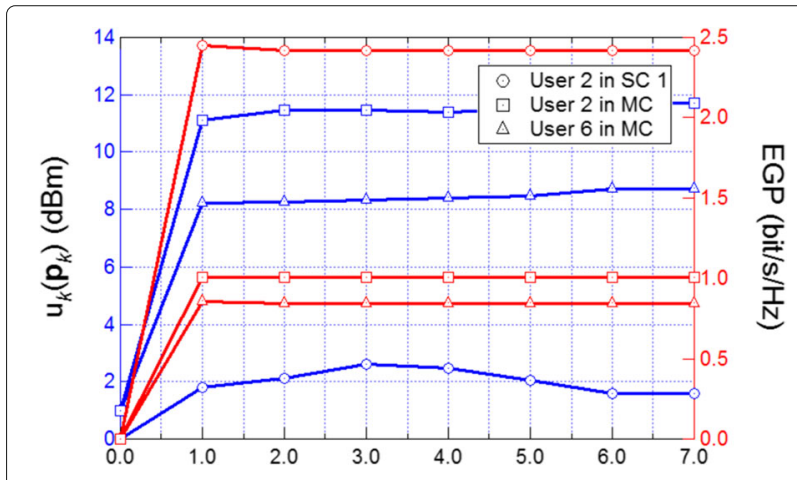

Fig. 7 Power and EGP convergence 
coverage area of the MC, Fig. 8 compares the average total transmitted power spent in the HetNet versus the distance from the selected users and their BS, i.e., user 1 in the SC 1 and user 1 in the MC. Two scenarios are addressed: together with the $\mathrm{MC}$, we consider the presence of $Q=1 \mathrm{SC}$ or $Q=4 \mathrm{SCs}$, being in both cases, for a fair comparison, the total number of active users in the HetNet equal to 18. Particularly, for $Q=1, K_{1}=3$ users are served within the SC 1 , and the 15 remaining ones belong to the MC. Conversely, for $Q=4$, according to the scenario of Fig. 5, three users are connected to each SBS, and only six to the MBS. For each distance, the total power is averaged over 100 independent transmitted packets and network geometry different realizations, whereas the minimum target EGP is set to $\bar{\zeta}=2 \mathrm{bit} / \mathrm{s} / \mathrm{Hz}$. As for Fig. 8, empty and solid square marks are used for the case of $Q=1 \mathrm{SC}$, whereas the curves with empty and solid circle marks refer to the case of $Q=4$ SCs. As expected from the working of the distributed RA algorithm, the total power spent in the HetNet grows with increasing the distance, due to the fact that the minimum target EGP has to be guaranteed while allocating the minimum power to each user. Further, when the number of SCs is increased moving from $Q=1$ to $Q=4$, the interference gets higher, though slightly enough, since the SCs are not packed together. But, more users are located closer to their BS, with the result that each one will require less power, and consequently, the total power spent within the HetNet will be lower as well. Therefore, the larger number of sparse SCs, the less power is required to meet the QoS constraints in spite of higher ICI, thus corroborating the advantages of the SC concept.

Finally, let us assess the scalability of the proposed distributed RA solution when many more BSs and users are present within the MC. To this end, Fig. 9 shows the new considered scenario called dense HetNet, which

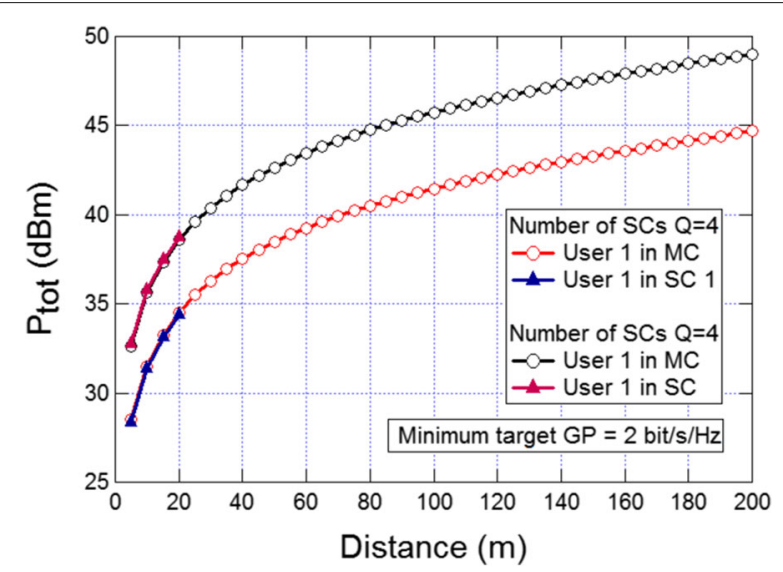

Fig. 8 Average total transmitted power of the HetNet vs. user distance from MBS or SBS

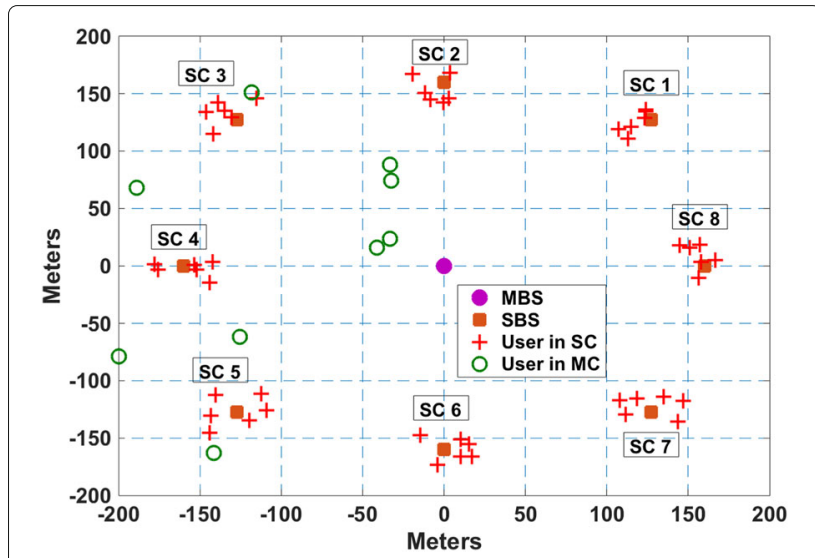

Fig. 9 Dense HetNet simulated scenario

is composed by a MC with eight users and eight SCs with six users for each of them, thus notably increasing the number of users and SCs compared to the scenario considered so far. On the basis of the new dense HetNet scenario, Fig. 10 depicts the convergence of power and EGP for the same users in the same geometric position of Fig. 7. Comparing results in Fig. 7 with those in Fig. 10, we point out that the algorithm is scalable since the fast convergence to the equilibrium point is still guaranteed.

\section{Conclusions}

This paper tackled the distributed RA problem, in the uplink of HetNets, aimed at minimizing the power consumption of each user under the satisfaction of a minimum GP constraint per user. The problem, tagged as PEGE OP, was faced by suitably decoupling the $\mathrm{BL}$ and coding rate allocation problem from the PA problem. First, the PEGE OP in P2P links was investigated and solved, obtaining in particular an approximate yet accurate closed-form expression of the PA, in a water-fillinglike form, which depends on the QoS constraint. Then,

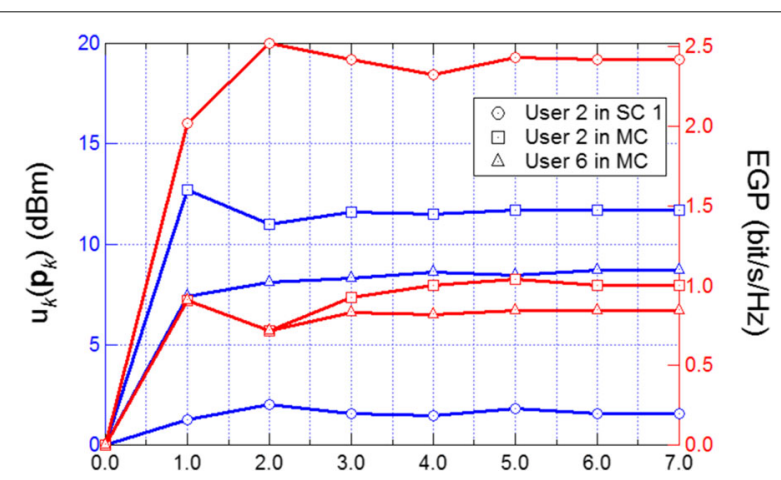

Fig. 10 Power and EGP convergence in dense HetNet 
we moved to the interference channel case, where the PA OP was described as a NCG. The relevant solution concept was identified in the GNE, due to QoS constraint that couples the strategies (i.e., the set of feasible PA vectors) of the players (i.e., the users in the HetNet transmitting over the same frequencies). Capitalizing on the closed-form expression for the PA, the analysis of the GNE was carried out through the BR concept, providing sufficient conditions for the existence and uniqueness of the solution. Finally, a distributed RA algorithm, which converges to the equilibrium of the game, was derived and its performance tested through extensive numerical simulations, certifying its good convergence properties, the reduction of power consumption in the HetNet, and the close match between EGP and AGP. Further line of research on this topic will be the analysis of the impact of the total power constraint on the equilibrium.

\section{Endnote}

${ }^{1}$ The BL solution found applying the algorithm in [37] clearly depends on the initial PA taken over the subcarriers. Assuming an uniform PA, however, allows to have initially all the subcarriers being active, thus, to perform BL over all the subcarriers. This approach is suboptimal yet simple and efficient, as shown by the result obtained in [31].

\section{Appendix A: Proof of Proposition 1}

Since both the objective function and the constraints are convex, (14) is a convex OP. Introducing the multipliers $\Theta$, $\boldsymbol{v} \triangleq\left[v_{1}, \cdots, v_{N}\right]^{T}$ and $\lambda \triangleq\left[\lambda_{1}, \cdots, \lambda_{N}\right]^{T}$, the associated Lagrangian is

$$
L(\mathbf{p}, \Theta, \boldsymbol{v})=\sum_{n=1}^{N} p_{n}+\Theta\left(\beta \sum_{n=1}^{N} \mathrm{e}^{-\frac{p_{n}}{\rho_{n}}}-\kappa\right)-\sum_{n=1}^{N} v_{n} p_{n}
$$

and, applying the KKT conditions, we have necessary and sufficient condition for optimality, i.e.,

$$
\begin{gathered}
1-\Theta^{*} \frac{\beta}{\rho_{n}} \mathrm{e}^{-\frac{p_{n}^{*}}{\rho_{n}}}-v_{n}^{*}=0, \quad \forall n \in \mathcal{N}, \\
v_{n}^{*} p_{n}^{*}=0, \forall n \in \mathcal{N}, \quad \Theta^{*}\left(\beta \sum_{n=1}^{N} \mathrm{e}^{-\frac{p_{n}^{*}}{\rho_{n}}}-\kappa\right)=0, \\
p_{n}^{*} \geq 0, \quad \forall n \in \mathcal{N}, \quad v_{n}^{*} \geq 0, \forall n \in \mathcal{N}, \quad \Theta^{*} \geq 0,
\end{gathered}
$$

where $\mathbf{p}^{*}$ and $\left\{\Theta^{*}, \boldsymbol{v}^{*}\right\}$ denote the optimal primal and dual variables, respectively. After some algebra, from (30) it easily follows that $p_{n}^{*}=\rho_{n}\left[\log \Theta^{*}-\log \frac{\rho_{n}}{\beta_{n}}\right]^{+}$, where $\Theta^{*}$ is such that the QoS constraint in (14) holds with equality.

\section{Appendix B: Proof of Proposition 3}

Sufficient conditions for the feasibility of (17), i.e., there is a non-empty solution set, are derived considering that the QoS constraint must hold true $\forall k \in \mathcal{K}$. This constraint can be equivalently rewritten as

$$
\sum_{n \in \mathcal{N}_{k}}\left(\beta_{k} \mathrm{e}^{-\frac{p_{k, n}}{\rho_{k, n}\left(\mathbf{p}_{-k, n}\right)}}-\frac{\kappa_{k}}{N_{k}}\right) \leq 0
$$

so that a more stringent condition, recalling that $\kappa_{k}=$ $m_{k} N_{k} \mathrm{e}^{-\gamma_{k}^{*}}$, is

$$
\beta_{k} \mathrm{e}^{-\frac{p_{k, n}}{\rho_{k, n}(\mathbf{p}-k, n)}} \leq \mathrm{e}^{-\gamma_{k}^{*}}, \quad \forall k, n .
$$

Exploiting the definition of $\rho_{k, n}$ in (18) and defining $\tilde{\gamma}_{k}^{*} \triangleq$ $\gamma_{k}^{*}+\log \beta_{k}>0$ (being $\beta_{k}>1$ ), after some algebra (32) becomes

$$
p_{k, n}\left|\bar{h}_{k, \phi(k), n}\right|^{2}-\tilde{\gamma}_{k}^{*} \sum_{\substack{j=1 \\ j \neq k}}^{K} p_{j, n}\left|h_{j, \phi(k), n}\right|^{2} \geq \tilde{\gamma}_{k}^{*} \sigma_{k, n}^{2}, \quad \forall k, n .
$$

The above expression can be recast into a matrix form as

$$
\mathbf{Z}_{n}\left(\boldsymbol{\gamma}^{*}\right) \cdot \mathbf{q}_{n} \geq \mathbf{t}_{n}\left(\boldsymbol{\gamma}^{*}\right) \geq \mathbf{0}, \quad \forall n,
$$

that is,

$$
\mathbf{q}_{n} \geq\left[\mathbf{Z}_{n}\left(\boldsymbol{\gamma}^{*}\right)\right]^{-1} \cdot \mathbf{t}_{n}\left(\boldsymbol{\gamma}^{*}\right) \geq \mathbf{0}, \quad \forall n,
$$

where $\mathbf{Z}_{n}$ and $\mathbf{t}_{n}$ are defined in (25) and (24), respectively, and $\mathbf{q}_{n} \triangleq\left[p_{1, n}, \cdots, p_{K, n}\right]^{\mathrm{T}}$.

Looking at the expression of $\mathbf{Z}_{n}$ in (25), it is a $Z$-matrix, since all its off-diagonal entries are negative. Then, if $\mathbf{Z}_{n}$ is also a $P$-matrix, its inverse is nonnegative [40] and thus, since $\mathbf{t}_{n} \geq \mathbf{0}$, condition (35) is satisfied, i.e., the problem is feasible since there exist at least one solution.

\section{Appendix C: Proof of Proposition 4}

According to the Nash existence theorem [41], given a game in strategic form with $K$ players, each characterized by an action space $\mathcal{P}_{k}$ and an utility function $u_{k}$, if, $\forall k \in \mathcal{K}$, (i) $\mathcal{P}_{k}$ is non-empty, convex and compact, (ii) $u_{k}: \mathcal{P} \rightarrow \mathbb{R}$ is continuous with $\mathcal{P} \triangleq \mathcal{P}_{1} \times \cdots \times \mathcal{P}_{K}$, and (iii) $\forall \mathbf{p}_{-k} \in \mathcal{P} \backslash \mathcal{P}_{k}, u_{k}$ is concave on $\mathcal{P}_{k}$, then a Nash equilibrium exists. Conditions (ii) and (iii) and the convexity and compactness of each $\mathcal{P}_{k}$ easily follow by looking at their analytical expression. A sufficient condition for the non-emptiness of the sets $\mathcal{P}_{k}, \forall k \in \mathcal{K}$, is given by the feasibility condition stated in Proposition 3 . This proves the first part of Proposition 4.

Under the feasibility condition, i.e., if $\mathbf{Z}_{n}$ is a $P$-matrix, then there exist at least $N K$-sized vectors $\mathbf{q}_{n}^{*} \triangleq$ $\left[p_{1, n}^{*}, \cdots, p_{K, n}^{*}\right]^{\mathrm{T}}, 1 \leq n \leq N$, which satisfy (26) in Appendix B and are thus the solution to OP (17). This proves the second part of Proposition 4. 


\section{Appendix D: Proof of Proposition 5}

The KKT conditions of OP (17) are

$$
\begin{gathered}
1-\Theta_{k} \frac{\beta_{k}}{\rho_{k, n}(\mathbf{p}-k, n)} \mathrm{e}^{-\frac{p_{k, n}}{\rho_{k, n}\left(\mathbf{p}_{-k, n}\right)}}-v_{k, n}=0, \quad \forall k, n, \\
v_{k, n} p_{k, n}=0, \forall k, n, \quad \Theta_{k}\left(\sum_{n \in \mathcal{N}_{k}} \beta_{k} \mathrm{e}^{\left.-\frac{p_{k, n}}{\rho_{k, n}(\mathbf{P}-k, n}\right)}-\kappa_{k}\right)=0, \quad \forall k \\
p_{k, n} \geq 0, \forall k, n, \quad v_{k, n} \geq 0, \forall k, n, \quad \Theta_{k} \geq 0, \quad \forall k .
\end{gathered}
$$

Eliminating the Lagrange multiplier $\left\{v_{k, n}\right\}$ relevant to the nonnegativity constraint of the power coefficients and making some substitutions (36) can be equivalently rewritten as the following mixed nonlinear complementary problem (MNCP):

$$
\begin{aligned}
& 0 \leq p_{k, n} \perp 1-\Theta_{k} \frac{\beta_{k}}{\rho_{k, n}\left(\mathbf{p}_{-k, n}\right)} \mathrm{e}^{-\frac{p_{k, n}}{\rho_{k, n}\left(\mathbf{p}_{-k, n}\right)}} \geq 0 \\
& 0 \leq \Theta_{k}, \quad \sum_{n \in \mathcal{N}_{k}} \beta_{k} \mathrm{e}^{-\frac{p_{k, n}}{\rho_{k, n}\left(\mathbf{p}_{-k, n}\right)}}=\kappa_{k} \quad \forall k .
\end{aligned}
$$

Let us now introduce the variable

$$
z_{k, n} \triangleq f\left(p_{k, n}\right)=1-\mathrm{e}^{-\frac{p_{k, n}}{\rho_{k, n}}}
$$

which is one-to-one related to $p_{k, n}$, i.e., $p_{k, n}=f^{-1}\left(z_{k, n}\right)$, and note that $z_{k, n}>0$ iff $p_{k, n}>0$ and $z_{k, n}=0$ iff $p_{k, n}=$ 0 . Thus, upon defining $\mathbf{z}_{-k, n} \triangleq f\left(\mathbf{p}_{-k, n}\right)$, the system of Eq. (37) can be rewritten as

$$
\begin{array}{rrr}
0 \leq z_{k, n} \perp & 1+\Theta_{k} \frac{\beta_{k}}{\rho_{k, n}\left(f\left(\mathbf{p}_{-k, n}\right)\right)}\left(z_{k, n}-1\right) & \geq 0 \forall k, n \\
0 \leq \Theta_{k}, & \sum_{n \in \mathcal{N}_{k}} \beta_{k}\left(1-z_{k, n}\right) & =\kappa_{k} \forall k,
\end{array}
$$

and after some algebra and taking the logarithm of the second inequality on the first line of (39), we get

$$
\begin{aligned}
& 0 \leq z_{k, n} \perp \log \frac{\rho_{k, n}\left(f\left(\mathbf{p}_{-k, n}\right)\right)}{\beta_{k}}+\lambda_{k}+\log \frac{1}{1-z_{k, n}} \geq 0 \quad \forall k, n \\
& \sum_{k} \text { free, } \quad z_{k \in \mathcal{N}_{k}}=\kappa_{k}^{\prime}
\end{aligned}
$$

with $\kappa_{k}^{\prime}=N_{k}-\kappa_{k} / \beta_{k}$. We now define the functions $J_{k, n}\left(z_{k, n}, \mathbf{z}_{-k, n}\right) \triangleq \log \frac{\rho_{k, n}\left(\mathbf{z}_{-k, n}\right)}{\beta_{k}}-\log \left(1-z_{k, n}\right)$, assumed continuously differentiable over the set $\prod_{k=1}^{K}\left[0, \kappa_{k}^{\prime}\right]$. Then, it follows that Eq. (40) is the KKT condition of the variational inequality $(\mathrm{VI})$ problem $\operatorname{VI}(U, \mathbf{J})$ [7], with $U \triangleq \prod_{k \in \mathcal{K}} U_{k}, U_{k} \triangleq\left\{\mathbf{z}_{k} \in \mathbb{R}^{N}: \sum_{n \in \mathcal{N}_{k}} z_{k, n}=\kappa_{k}^{\prime}\right\}$, $\mathbf{z}_{k} \triangleq\left[z_{k, 1}, \cdots, z_{k, N}\right]^{\mathrm{T}}, \quad \mathbf{J} \triangleq\left[\mathbf{J}_{1}^{T}, \cdots, \mathbf{J}_{K}^{\mathrm{T}}\right]$ and $\mathbf{J}_{k} \triangleq$ $\left[J_{k, 1}, \cdots, J_{k, N}\right]^{\mathrm{T}}$.

Thus, by definition, $\mathbf{z}_{k}^{\star}, \forall k$, is a solution of the $\operatorname{VI}(U, \mathbf{J})$, if and only if, $\forall \mathbf{z}_{k} \in U_{k}$, the following condition holds:

$$
\sum_{n \in \mathcal{N}_{k}}\left(z_{k, n}-z_{k, n}^{\star}\right)\left(\log \frac{\rho_{k, n}}{\beta_{k}}+\log \frac{1}{1-z_{k, n}^{\star}}\right) \geq 0 .
$$

Assume now there exist two solutions $\left\{\mathbf{p}_{k}^{(1)}\right\}\left(\left\{\mathbf{z}_{k}^{(1)}\right\}\right)$ and $\left\{\mathbf{p}_{k}^{(2)}\right\}\left(\left\{\mathbf{z}_{k}^{(1)}\right\}\right)$ of the GNE problem and be $\rho_{k, n}^{(i)} \triangleq$ $\rho_{k, n}\left(\mathbf{p}_{-k, n}^{(i)}\right), i=1,2$. From (41), it follows that they must satisfy

$$
\sum_{n \in \mathcal{N}_{k}}\left(z_{k, n}^{(2)}-z_{k, n}^{(1)}\right)\left(\log \frac{\rho_{k, n}^{(1)}}{\beta_{k}}+\log \frac{1}{1-z_{k, n}^{(1)}}\right) \geq 0,
$$

and

$$
\sum_{n \in \mathcal{N}_{k}}\left(z_{k, n}^{(1)}-z_{k, n}^{(2)}\right)\left(\log \frac{\rho_{k, n}^{(2)}}{\beta_{k}}+\log \frac{1}{1-z_{k, n}^{(2)}}\right) \geq 0 .
$$

Before proceeding further, let us introduce some definitions and inequalities that will be instrumental for the subsequent analysis:

$$
\begin{aligned}
\tau_{k, n}\left(\mathbf{p}_{-k, n}^{(i)}\right) & =\tau_{k, n}^{(i)} \triangleq \frac{\rho_{k, n}^{(i)}}{\left|h_{k, \phi(k), n}\right|^{2}} \\
& =\delta_{k}\left[\sigma_{k, n}^{2}+\sum_{\substack{j=1 \\
j \neq k}}^{K} p_{j, n}\left|h_{j, \phi(k), n}\right|^{2}\right] i=1,2,
\end{aligned}
$$

$$
\delta_{k} \sigma_{k, n}^{2} \leq \tau_{k, n}\left(\mathbf{p}_{-k, n}^{(i)}\right) \leq \bar{\tau}_{k, n} \triangleq \tau_{k, n}\left(\overline{\mathbf{p}}_{-k, n}\right) \quad i=1,2
$$

$$
\begin{aligned}
\frac{p_{k, n}^{(i)}}{\rho_{k, n}^{(i)}}= & \frac{p_{k, n}^{(i)}\left|h_{k, \phi(k), n}\right|^{2}}{\tau_{k, n}^{(i)}} \leq \frac{p_{k, n}^{(i)}\left|h_{k, \phi(k), n}\right|^{2}}{\sigma_{k, n}^{2}} \leq \frac{\bar{p}_{k, n}\left|h_{k, \phi(k), n}\right|^{2}}{\sigma_{k, n}^{2}} \\
& \leq \max _{n} \frac{\bar{p}_{k, n}\left|h_{k, \phi(k), n}\right|^{2}}{\sigma_{k, n}^{2}} \triangleq \bar{\gamma}_{k}^{(\max )} \quad i=1,2,
\end{aligned}
$$

where $\left\{\bar{p}_{k, n}\right\}$ coefficients are given by (26).

Summing (42) and (43), and after some simple algebra, we get

$$
\begin{gathered}
\sum_{n \in \mathcal{N}_{k}}\left(z_{k, n}^{(2)}-z_{k, n}^{(1)}\right)\left(\log \left(1-z_{k, n}^{(1)}\right)-\log \left(1-z_{k, n}^{(2)}\right)\right) \leq \\
\sum_{n \in \mathcal{N}_{k}}\left(z_{k, n}^{(2)}-z_{k, n}^{(1)}\right)\left(\log \rho_{k, n}^{(1)}-\log \rho_{k, n}^{(1)}\right) .
\end{gathered}
$$

Let us now recall the mean value theorem: if a function $g$ is continuous on the closed interval $[a, b]$, where $a<b$, and differentiable on the open interval $(a, b)$, then there exists a point $c$ in $(a, b)$ such that $g(b)-g(a)=g^{\prime}(c)(b-a)$. Accordingly, taking $g(\cdot)=\log (\cdot)$, we get 
$\log \left(1-z_{k, n}^{(1)}\right)-\log \left(1-z_{k, n}^{(2)}\right)=\frac{z_{k, n}^{(2)}-z_{k, n}^{(1)}}{s_{k, n}}, \mathrm{e}^{-\frac{p_{k, n}^{(2)}}{\rho_{k, n}^{(2)}}} \leq s_{k, n} \leq \mathrm{e}^{-\frac{p_{k, n}^{(1)}}{\rho_{k, n}^{(1)}}}$.

Substituting the above into (47), we get

$$
\begin{aligned}
\sum_{n \in \mathcal{N}_{k}}\left(z_{k, n}^{(2)}-z_{k, n}^{(1)}\right)^{2} & \leq \sum_{n \in \mathcal{N}_{k}} \frac{\left(z_{k, n}^{(2)}-z_{k, n}^{(1)}\right)^{2}}{s_{k, n}} \leq \sum_{n \in \mathcal{N}_{k}}\left(z_{k, n}^{(2)}-z_{k, n}^{(1)}\right) \\
& \times\left(\log \rho_{k, n}^{(1)}-\log \rho_{k, n}^{(2)}\right), \\
& \leq \sqrt{\sum_{n \in \mathcal{N}_{k}}\left(z_{k, n}^{(2)}-z_{k, n}^{(1)}\right)^{2}} \sqrt{\sum_{n \in \mathcal{N}_{k}}\left(\log \rho_{k, n}^{(1)}-\log \rho_{k, n}^{(2)}\right)^{2}},
\end{aligned}
$$

where the first inequality comes from the upper bound on $s_{k, n} \leq 1$ (which in turn can be inferred by recalling the bound in (48) and $\left.\mathrm{e}^{-p_{k, n}^{(i)} / \rho_{k, n}^{(i)}} \leq 1, \forall i, k, n\right)$, whereas the latter inequality comes from the triangle inequality. Thus, (49) implies

$$
\sqrt{\sum_{n \in \mathcal{N}_{k}}\left(z_{k, n}^{(2)}-z_{k, n}^{(1)}\right)^{2}} \leq \sqrt{\sum_{n \in \mathcal{N}_{k}}\left(\log \rho_{k, n}^{(1)}-\log \rho_{k, n}^{(2)}\right)^{2}} .
$$

According to (38), $z_{k, n}^{(2)}-z_{k, n}^{(1)}=\mathrm{e}^{-{\frac{p_{k, n}^{(1)}}{\rho_{k, n}^{(1)}}}^{(1)}}-\mathrm{e}^{-\frac{p_{k, n}^{(2)}}{\rho_{k, n}^{(2)}}}$, and exploiting the mean value theorem for the exponential function, we get

$$
\mathrm{e}^{-\frac{p_{k, n}^{(1)}}{\rho_{k, n}^{(1)}}}-e^{-\frac{p_{k, n}^{(2)}}{\rho_{k, n}^{(2)}}}=\mathrm{e}^{-w_{k, n}}\left(\frac{p_{k, n}^{(2)}}{\rho_{k, n}^{(2)}}-\frac{p_{k, n}^{(1)}}{\rho_{k, n}^{(1)}}\right), \frac{p_{k, n}^{(1)}}{\rho_{k, n}^{(1)}} \leq w_{k, n} \leq \frac{p_{k, n}^{(2)}}{\rho_{k, n}^{(2)}} .
$$

Moreover, by (46), $e^{-w_{k, n}} \geq e^{-\bar{\gamma}_{k}^{(\max )}}$, and exploiting relation (44), (50) becomes

$\mathrm{e}^{-\bar{\gamma}_{k}^{(\max )}} \sqrt{\sum_{n \in \mathcal{N}_{k}}\left(\frac{p_{k, n}^{(2)}}{\rho_{k, n}^{(2)}}-\frac{p_{k, n}^{(1)}}{\rho_{k, n}^{(1)}}\right)^{2}} \leq \sqrt{\sum_{n \in \mathcal{N}_{k}}\left(\log \tau_{k, n}^{(1)}-\log \tau_{k, n}^{(2)}\right)^{2}}$.

We now introduce the following definition

$$
\xi_{k, n} \triangleq\left(p_{k, n}^{(2)}-p_{k, n}^{(1)}\right)\left|h_{k, \phi(k), n}\right|^{2}
$$

and note that

$$
\begin{aligned}
\frac{p_{k, n}^{(2)}}{\rho_{k, n}^{(2)}}-\frac{p_{k, n}^{(1)}}{\rho_{k, n}^{(1)}}= & \left(\frac{p_{k, n}^{(2)}}{\tau_{k, n}^{(2)}}-\frac{p_{k, n}^{(1)}}{\tau_{k, n}^{(1)}}\right)\left|h_{k, \phi(k), n}\right|^{2} \\
= & \frac{p_{k, n}^{(2)}-p_{k, n}^{(1)}}{\tau_{k, n}^{(2)}}\left|h_{k, \phi(k), n}\right|^{2} \\
& +p_{k, n}^{(1)}\left|h_{k, \phi(k), n}\right|^{2}\left(\frac{1}{\tau_{k, n}^{(2)}}-\frac{1}{\tau_{k, n}^{(1)}}\right) \\
= & \frac{\xi_{k, n}}{\tau_{k, n}^{(2)}}-\frac{\tau_{k, n}^{(2)}-\tau_{k, n}^{(1)}}{\tau_{k, n}^{(2)}} \frac{\left|h_{k, \phi(k), n}\right|^{2} p_{k, n}^{(1)}}{\tau_{k, n}^{(1)}} .
\end{aligned}
$$

Accordingly, by noting that, due to the triangle inequality,

$$
\begin{aligned}
& \mathrm{e}^{-\bar{\gamma}_{k}^{(\max )}} \sqrt{\sum_{n \in \mathcal{N}_{k}}\left(\frac{p_{k, n}^{(2)}}{\rho_{k, n}^{(2)}}-\frac{p_{k, n}^{(1)}}{\rho_{k, n}^{(1)}}\right)^{2}} \\
&= \mathrm{e}^{-\bar{\gamma}_{k}^{(\max )}} \sqrt{\sum_{n \in \mathcal{N}_{k}}\left(\frac{\xi_{k, n}}{\tau_{k, n}^{(2)}}-\frac{\tau_{k, n}^{(2)}-\tau_{k, n}^{(1)}}{\tau_{k, n}^{(2)}} \frac{\left.h_{k, \phi(k), n}\right|^{2} p_{k, n}^{(1)}}{\tau_{k, n}^{(1)}}\right)^{2}} \\
& \geq \mathrm{e}^{-\bar{\gamma}_{k}^{(\max )}} \sqrt{\sum_{n \in \mathcal{N}_{k}}\left(\frac{\xi_{k, n}}{\tau_{k, n}^{(2)}}\right)^{2}} \\
&-\mathrm{e}^{-\bar{\gamma}_{k}^{(\max )}} \sqrt{\sum_{n \in \mathcal{N}_{k}}\left(\frac{\tau_{k, n}^{(2)}-\tau_{k, n}^{(1)}}{\tau_{k, n}^{(2)}} \frac{\left|h_{k, \phi(k), n}\right|^{2} p_{k, n}^{(1)}}{\tau_{k, n}^{(1)}}\right)^{2}},
\end{aligned}
$$

and that, by the mean value theorem of the logarithmic function,

$$
\log \left(\tau_{k, n}^{(1)}\right)-\log \left(\tau_{k, n}^{(2)}\right)=\frac{\tau_{k, n}^{(1)}-\tau_{k, n}^{(2)}}{t_{k, n}}, \tau_{k, n}^{(1)} \leq t_{k, n} \leq \tau_{k, n}^{(2)},
$$

we can bound (52) as follows

$$
\begin{aligned}
\mathrm{e}^{-\bar{\gamma}_{k}^{(\max )}} & \sqrt{\sum_{n \in \mathcal{N}_{k}}\left(\frac{\xi_{k, n}}{\tau_{k, n}^{(2)}}\right)^{2}} \\
\leq & \mathrm{e}^{-\bar{\gamma}_{k}^{(\max )}} \sqrt{\sum_{n \in \mathcal{N}_{k}}\left(\frac{\tau_{k, n}^{(2)}-\tau_{k, n}^{(1)}}{\tau_{k, n}^{(2)}} \frac{\left|h_{k, \phi(k), n}\right|^{2} p_{k, n}^{(1)}}{\tau_{k, n}^{(1)}}\right)^{2}}, \\
& +\sqrt{\sum_{n \in \mathcal{N}_{k}}\left(\frac{\tau_{k, n}^{(1)}-\tau_{k, n}^{(2)}}{t_{k, n}}\right)^{2}},
\end{aligned}
$$

$$
\leq \sqrt{\sum_{n \in \mathcal{N}_{k}}\left(\frac{\tau_{k, n}^{(2)}-\tau_{k, n}^{(1)}}{\tau_{k, n}^{(2)}} \bar{\gamma}_{k}^{(\max )}\right)^{2}}+\sqrt{\sum_{n \in \mathcal{N}_{k}}\left(\frac{\tau_{k, n}^{(2)}-\tau_{k, n}^{(1)}}{t_{k, n}}\right)^{2}},
$$




$$
\begin{aligned}
& \leq\left(\bar{\gamma}_{k}^{(\max )}+1\right) \sqrt{\sum_{n \in \mathcal{N}_{k}}\left(\frac{\tau_{k, n}^{(2)}-\tau_{k, n}^{(1)}}{\delta_{k} \sigma_{k, n}^{2}}\right)^{2}} \\
& \leq \mathrm{e}^{\bar{\gamma}_{k}^{(\max )}} \sqrt{\sum_{n \in \mathcal{N}_{k}}\left(\sum_{\substack{j=1 \\
j \neq k}}^{K} \frac{\left(p_{j, n}^{(2)}-p_{j, n}^{(1)}\right)\left|h_{j, \phi(k), n}\right|^{2}}{\sigma_{k, n}^{2}}\right)^{2}},
\end{aligned}
$$

where in (58) we exploited the fact that $\mathrm{e}^{-x} \leq 1, \forall x \geq 0$ and (46), in (59) the lower bound in (45) and in (60) the bound $x+1 \leq \mathrm{e}^{x}, \forall x \geq 0$. By exploiting the upper bound in (45), and defining

$$
\chi_{j, k, n} \triangleq \frac{\bar{\tau}_{j, n}}{\sigma_{k, n}^{2}} \frac{\left|h_{j, \phi(k), n}\right|^{2}}{\left|h_{j, \phi(j), n}\right|^{2}},
$$

inequality (60) can be further rearranged as

$$
\mathrm{e}^{-\bar{\gamma}_{k}^{(\max )}} \sqrt{\sum_{n \in \mathcal{N}_{k}}\left(\frac{\xi_{k, n}}{\bar{\tau}_{k, n}}\right)^{2}} \leq \mathrm{e}^{\bar{\gamma}_{k}^{(\max )}} \sqrt{\sum_{n \in \mathcal{N}_{k}}\left(\sum_{\substack{j=1 \\ j \neq k}}^{K} \chi_{j, k, n} \frac{\xi_{j, n}}{\bar{\tau}_{j, n}}\right)^{2}}
$$

$$
\leq \mathrm{e}^{\bar{\gamma}_{k}^{(\max )}} \sum_{\substack{j=1 \\ j \neq k}}^{K}\left(\max _{n} \chi_{j, k, n}\right) \sqrt{\sum_{n \in \mathcal{N}_{k}}\left(\frac{\xi_{j, n}}{\bar{\tau}_{j, n}}\right)^{2}} .
$$

Hence, by defining

$$
\chi_{j, k}^{(\max )} \triangleq \max _{n}\left(\chi_{j, k, n}\right)
$$

expression (63) can be rewritten in matrix form as

$$
\mathbf{V b} \leq \mathbf{0},
$$

where

$\mathbf{V}=\left[\begin{array}{cccc}\mathrm{e}^{-\bar{\gamma}_{1}^{(\max )}} & -\mathrm{e}^{\bar{\gamma}_{1}^{(\max )}} \chi_{2,1}^{(\max )} & \cdots & -\mathrm{e}^{\bar{\gamma}_{1}^{(\max )}} \chi_{K, 1}^{(\max )} \\ -\mathrm{e}^{\bar{\gamma}_{2}^{(\max )}} \chi_{1,2}^{(\max )} & \mathrm{e}^{-\bar{\gamma}_{2}^{(\max )}} & \cdots & -\mathrm{e}^{\bar{\gamma}_{2}^{(\max )}} \chi_{K, 2}^{(\max )} \\ \vdots & \vdots & \ddots & \vdots \\ -\mathrm{e}^{\bar{\gamma}_{K}^{(\max )}} \chi_{1, K}^{(\max )} & -\mathrm{e}^{\bar{\gamma}_{K}^{(\max )}} \chi_{2, K}^{(\max )} & \cdots & \mathrm{e}^{-\bar{\gamma}_{K}^{(\max )}}\end{array}\right]$

and

$$
\mathbf{b} \triangleq\left[\begin{array}{c}
\sqrt{\sum_{n \in \mathcal{N}_{1}}\left(\frac{\xi_{1, n}}{\bar{\tau}_{1, n}}\right)^{2}} \\
\vdots \\
\sqrt{\sum_{n \in \mathcal{N}_{K}}\left(\frac{\xi_{K, n}}{\bar{\tau}_{K, n}}\right)^{2}}
\end{array}\right] .
$$

It can be noted that $\mathbf{V}$ is a $Z$-matrix. Then, if $\mathbf{V}$ is a $P$ matrix, it must have a nonnegative inverse, implying $\mathbf{b}=$ 0 . If $\mathbf{b}=0$, then the equilibrium is unique. This proves Proposition 4.

\section{Acknowledgements}

This work has been partially supported by the PRA 2016 research project 5 GIOTTO funded by the University of Pisa.

\section{Competing interests}

The authors declare that they have no competing interests.

Received: 1 April 2016 Accepted: 23 November 2016

Published online: 01 December 2016

\section{References}

1. Ericsson, $5 G$ Radio Access - Capabilities and technologies. Ericsson White Paper, 1-10 (2016). https://www.ericsson.com/res/docs/whitepapers/ wp-5g.pdf

2. The Climate Group and Global e-Sustainability Initiative (GeSI), 2008. SMART 2020: enabling the low carbon economy in the information age. http://gesi.org/article/43. Accessed 2008

3. X Sun, S Wang, Resource allocation scheme for energy saving in heterogeneous networks. Wirel. Commun. IEEE Trans. 14(8), 4407-4416 (2015)

4. J Hoydis, M Kobayashi, M Debbah, Green small-cell networks. Veh. Technol. Mag. IEEE. 6(1), 37-43 (2011)

5. N Saquib, E Hossain, LB Le, DI Kim, Interference management in OFDMA femtocell networks: issues and approaches. Wirel. Commun. IEEE. 19(3), 86-95 (2012)

6. G Bacci, EV Belmega, P Mertikopoulos, L Sanguinetti, Energy-aware competitive power allocation for heterogeneous networks under QoS constraints. IEEE Trans. Wirel. Commun. 14(9), 4728-4742 (2015)

7. G Scutari, D Palomar, F Facchinei, J-S Pang, Convex optimization, game theory, and variational inequality theory. IEEE Signal Process. Mag. 27(3), 35-49 (2010)

8. J Chen, Y Zhou, Y Kuo, Energy-efficiency resource allocation for cognitive heterogeneous networks with imperfect channel state information. IET Commun. 10(11), 1312-1319 (2016)

9. J Ding, L Jiang, $\mathrm{CHe}$, Joint energy-efficient resource allocation and interference alignment for hetnets. IET Commun. 10(9), 1104-1110 (2016)

10. N Mokari, F Alavi, S Parsaeefard, T Le-Ngoc, Limited-feedback resource allocation in heterogeneous cellular networks. IEEE Trans. Veh. Technol. 65(4), 2509-2521 (2016)

11. TE Bogale, LB Le, Massive mimo and mmwave for $5 \mathrm{~g}$ wireless hetnet: potential benefits and challenges. IEEE Veh. Technol. Mag. 11(1), 64-75 (2016)

12. D Fudenberg, J Tirole, Game Theory. (MIT Press, Cambridge, 1991)

13. $\mathrm{H} \mathrm{Ji}, \mathrm{C}-\mathrm{Y}$ Huang, Non-cooperative uplink power control in cellular radio systems. Wirel. Netw. 4(3), 233-240 (1998)

14. W Yu, G Ginis, JM Cioffi, Distributed multiuser power control for digital subscriber lines. IEEE J. Sel. Areas Commun. 20(5), 1105-1115 (2002)

15. CU Saraydar, NB Mandayam, DJ Goodman, Efficient power control via pricing in wireless data networks. Trans. Commun. IEEE. 50(2), 291-303 (2002)

16. F Meshkati, SC Poor, HV Schwartz, NB Mandayam, An energy-efficient approach to power control and receiver design in wireless data networks. Trans. Commun. IEEE. 53(11), 1885-1894 (2005)

17. G Miao, N Himayat, GY Li, S Talwar, Distributed interference-aware energy-efficient power optimization. IEEE Trans. Wirel. Commun. 10(4), $1323-1333$ (2011) 
18. Y Kwon, T Hwang, X Wang, Energy-efficient transmit power control for multi-tier MIMO HetNets. IEEE J. Sel. Areas Commun. 33(10), 2070-2086 (2015)

19. Z Tian, G Leus, V Lottici, Joint dynamic resource allocation and waveform adaptation for cognitive networks. IEEE J. Selected Areas Commun. 29(2), 443-454 (2011)

20. G Scutari, DP Palomar, S Barbarossa, Optimal linear precoding strategies for wideband non-cooperative systems based on game theory-Part l: Nash equilibria. IEEE Trans. Signal Process. 56(3), 1230-1249 (2008)

21. G Scutari, DP Palomar, S Barbarossa, Optimal linear precoding strategies for wideband non-cooperative systems based on game theory - Part II: algorithms. IEEE Trans. Signal Process. 56(3), 1250-1267 (2008)

22. G Scutari, DP Palomar, S Barbarossa, Competitive design of multiuser MIMO systems based on game theory: a unified view. IEEE J. Sel. Areas Commun. 26(7), 1089-1103 (2008)

23. J-S Pang, G Scutari, F Facchinei, C Wang, Distributed power allocation with rate constraints in Gaussian parallel interference channels. IEEE Trans. Inf. Theory. 54(8), 3471-3489 (2008)

24. W Zhong, Y Fang, S Jin, KK Wong, S Zhong, Z Qian, Joint resource allocation for device-to-device communications underlaying uplink mimo cellular networks. IEEE J. Sel. Areas Commun. 33(1), 41-54 (2015)

25. W Zhong, B Fang, Z Qian, Game theoretic resource allocation for $\mathrm{d} 2 \mathrm{~d}$ mimo heterogeneous networks. Wireless Pers. Commun. 84, 273-285 (2015)

26. L Xiao, M Johansson, SP Boyd, Simultaneous routing and resource allocation via dual decomposition. IEEE Trans. Commun. 52(7), 1136-1144 (2004)

27. D Qiao, S Choi, KG Shin, Goodput analysis and link adaptation for IEEE 802.11 a wireless LANs. IEEE Trans. Mobile Comput. 1(4), 278-292 (2002)

28. T Kastrinogiannis, V Karyotis, S Papavassiliou, in Sarnoff Symposium, 2008 IEEE, Princeton, NJ, USA. An opportunistic combined power and rate allocation approach in cdma ad hoc networks (IEEE, New Jersey, 2008), pp. 1-5

29. A Giovanidis, S Stanczak, Stability and distributed power control in manets with per hop retransmissions. IEEE Trans. Commun. 59(6), 1632-1643 (2011)

30. YW Blankenship, PJ Sartori, BK Classon, V Desai, KL Baum, in IEEE 60th Vehicular Tech. Conf., 2004. VTC2004-Fall, Los Angeles, USA. Link error prediction methods for multicarrier systems, vol. 6 (IEEE, Los Angeles, 2004), pp. 4175-4179

31. I Stupia, V Lottici, F Giannetti, L Vandendorpe, Link resource adaptation for multiantenna bit-interleaved coded multicarrier systems. IEEE Trans. Signal Process. 60(7), 3644-3656 (2012)

32. F Facchinei, C Kanzow, Generalized Nash equilibrium problems. 4OR. 5(3), 173-210 (2007)

33. L Song, NB Mandayam, Hierarchical SIR and rate control on the forward link for CDMA data users under delay and error constraints. J. Sel. Areas Commun. 19(10), 1871-1882 (2001)

34. H Seo, BG Lee, Optimal transmission power for single- and multi-hop links in wireless packet networks with ARQ capability. IEEE Trans. Commun. 55(5), 996-1006 (2007)

35. MJ Osborne, A Rubinstein, A Course in Game Theory. (The MIT Press, Cambridge, 1994)

36. S Boyd, L Vandenberghe, Convex Optimization. (Cambridge Univ. Press, Cambridge, 2004)

37. I Stupia, F Giannetti, V Lottici, R Andreotti, L Vandendorpe, AN D'Andrea, in Future Network and Mobile Summit (FUNEMS): 16-18 June 2010; Florence, Italy. A greedy algorithm for goodput-oriented amc in turbo-coded ofdm, (2010), pp. 1-8

38. RA Horn, Matrix Analysis. (CR Johnson, ed.) (Cambridge University Press, New York, 1986)

39. G Calcev, D Chizhik, B Goransson, S Howard, H Huang, A Kogiantis, AF Molisch, AL Moustakas, D Reed, HXu, A wideband spatial channel model for system-wide simulations. IEEE Trans. Veh. Technol. 56(2), 389-403 (2007)
40. (RW Cottle, J-S Pang, RE Stone, eds.), The Linear Complementarity Problem. (SIAM, Academic Press, Philadelphia, 2009)

41. C Dutang, Existence theorems for generalized Nash equilibrium problems: an analysis of assumptions. HAL archives-ouvertes (2013). https://hal.archives-ouvertes.fr/hal-00828948/document

\section{Submit your manuscript to a SpringerOpen ${ }^{\circ}$ journal and benefit from:}

- Convenient online submission

Rigorous peer review

- Immediate publication on acceptance

- Open access: articles freely available online

- High visibility within the field

- Retaining the copyright to your article

Submit your next manuscript at $\gg$ springeropen.com 